\title{
The Bologna complete sample of nearby radio sources
}

\section{Phase referenced observations of faint nuclear sources $\star$}

\author{
E. Liuzzo ${ }^{1,2}$, G. Giovannini ${ }^{1,2}$, M. Giroletti ${ }^{1}$, and G. B. Taylor ${ }^{3,4}$ \\ 1 INAF Istituto di Radioastronomia, via Gobetti 101, 40129 Bologna, Italy \\ e-mail: ggiovann@ira.inaf.it \\ 2 Dipartimento di Astronomia, Università di Bologna, via Ranzani 1, 40127 Bologna, Italy \\ 3 Department of Physics and Astronomy, University of New Mexico, Albuquerque NM 87131, USA \\ 4 also Adjunct Astronomer at the National Radio Astronomy Observatory, USA \\ Received 27 May 2009 / Accepted 30 June 2009
}

ABSTRACT

\begin{abstract}
Aims. To study statistical properties of different classes of sources, it is necessary to observe a sample that is free of selection effects. To do this, we initiated a project to observe a complete sample of radio galaxies selected from the B2 Catalogue of Radio Sources and the Third Cambridge Revised Catalogue (3CR), with no selection constraint on the nuclear properties. We named this sample "the Bologna Complete Sample" (BCS).

Methods. We present new VLBI observations at 5 and $1.6 \mathrm{GHz}$ for 33 sources drawn from a sample not biased toward orientation. By combining these data with those in the literature, information on the parsec-scale morphology is available for a total of 76 of 94 radio sources with a range in radio power and kiloparsec-scale morphologies.

Results. The fraction of two-sided sources at milliarcsecond resolution is high (30\%), compared to the fraction found in VLBI surveys selected at centimeter wavelengths, as expected from the predictions of unified models. The parsec-scale jets are generally found to be straight and to line up with the kiloparsec-scale jets. A few peculiar sources are discussed in detail.
\end{abstract}

Key words. galaxies: active - galaxies: jets - galaxies: nuclei - radio continuum: galaxies

\section{Introduction}

The study of the parsec scale properties of radio galaxies is crucial for obtaining information on the nature of their central engine. To study the statistical properties of different classes of sources, it is necessary to define and observe a sample that is free of selection effects. To this aim, it is important to select samples using low radio frequencies. Sources in low-frequency samples are dominated by their extended and unbeamed (isotropic) emission, rather than the beamed compact emission that dominates in high-frequency surveys. Low frequency surveys are therefore unbiased with respect to the orientation of the nuclear relativistic jet. With this purpose in mind, we initiated a project to observe a complete sample of radio galaxies selected from the B2 Catalogue of Radio Sources and the Third Cambridge Revised Catalogue (3CR) (Giovannini et al. 1990, 2001), with no selection constraint on the nuclear properties. We named this sample "the Bologna Complete Sample" (BCS).

In the original sample, 95 radio sources from the B2 and $3 \mathrm{CR}$ catalogues were present, but because of the rejection of one source discussed here, we redefined the complete sample to be 94 sources. We selected the sources to be stronger than a flux density limit of $0.25 \mathrm{Jy}$ at $408 \mathrm{MHz}$ for the B2 sources and greater than $10 \mathrm{Jy}$ at $178 \mathrm{MHz}$ for the 3CR sources (Feretti et al. 1984) and applied the following criteria:

1) declination $>10^{\circ}$;

2) Galactic latitude $|b|>15^{\circ}$;

3) redshift $z<0.1$.

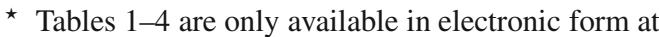
http://www . aanda. org
In Paper I (Giovannini et al. 2005), we presented the sample and discussed VLBI observations for an initial group of 53 sources having bright nuclear emission. Observations were carried on with the standard VLBI technique. We found that:

- no intrinsic difference in apparent jet speed and morphology on the parsec scale has been found between high and low power radio galaxies. A one-sided jet morphology is the predominant structure on the parsec scale, however $\sim 30 \%$ of the observed sources show evidence of a two-sided structure which has been quite rare in previous observations of the sources with high-power cores. This result is in rough agreement with a random orientation of radio galaxies and a high jet velocity $(\beta \sim 0.9)$;

- with very few exceptions, the parsec and the kiloparsecscale radio structures are aligned confirming that the large bends present in some BL-Lacs are likely amplified by their small angles between the jet direction and the line-of-sight. In sources with aligned parsec and kiloparsec scale structure, the main jet is always on the same side as the parsec-scale emission;

- in $\sim 30 \%$ of the sources, the correlated VLBI flux density is less than $70 \%$ of the arcsecond core flux density at the same frequency suggesting the presence of sub-kiloparsecscale structures;

- no polarized flux has been detected, confirming the low level of polarized emission in radio galaxies at parsec resolution.

Moreover a few sources have been discussed in detail and results have been presented in several papers (references are provided to these in Table 1).

Here we present new VLBA observations at $5 \mathrm{GHz}$ for 26 radio galaxies with a core flux density $S_{5 \mathrm{GHz}}>5 \mathrm{mJy}, 23$ of them 
observed for the first time at mas resolution. We also present new VLBA observations at $1.6 \mathrm{GHz}$ for 10 radio galaxies in order to study their extended nuclear emission. Since a few sources were observed at both frequencies, the total number of sources with new data presented here is 33 . For all sources we report on parsec-scale observations and discuss in detail their structure and properties.

To complete VLBI observations of the BCS sample, 18 sources of the sample will be observed with VLBI in the future. Since these sources have a very faint nuclear emission in the radio band we will need very sensitive VLBI observations (large bandwidths, long integration times, and phase referencing).

We assume here a Hubble constant $H_{0}=70 \mathrm{~km} \mathrm{~s}^{-1} \mathrm{Mpc}^{-1}$, $\Omega_{\mathrm{M}}=0.3$ and $\Omega_{\lambda}=0.7$.

\section{The sample}

In Table 1, we give the complete list of radio sources and summarize the most relevant information. We reported the IAU name (Col. 1), other name (Col. 2) and the redshift of the sources (Col. 3). For the kiloparsec morphology, we indicate if the source is a Faranoff types I or II radio galaxy (FRI/II), or a compact source, further distinguishing between flat spectrum compact sources (C), and Compact Steep Spectrum sources (CSS). Among flat spectrum compact sources, two are identified with BL-Lac type objects. Since these are well known objects (Mkn 421 and Mkn 501), in Table 1 and in the text we refer to them as BL-Lac objects. We indicate with $S_{\mathrm{c}, 5}$ the arcsecond core flux density at $5 \mathrm{GHz}$ and with $\log P_{\mathrm{c}}$ the corresponding logarithm of the radio power. $\log P_{\mathrm{t}}$ is the total radio power at $408 \mathrm{MHz}$. Notes in the last column refer to the status of VLBI observations: (N) new VLBI data are given for the first time in this paper, $\left(\mathrm{N}^{*}\right)$ the source was previously observed with VLBI, but new data are presented here, (G) see Giovannini et al. (2001), (I) see Paper I (Giovannini et al. 2005). Numbers refer to recent (post 2001) papers where a few peculiar sources have been discussed in detail.

For all sources, high-quality images at arcsecond resolution obtained with the Very Large Array (VLA) of the $\mathrm{NRAO}^{1}$ are available in the literature, allowing a detailed study of the largescale structure. According to the kiloparsec scale morphology, the sample contains 65 FRI radio galaxies, 16 FRII sources, and 13 compact sources. According to the optical and large-scale radio structure, one source $(0722+30)$ has been classified as a spiral galaxy and therefore should not be included in this sample. Because of its peculiarity it will be discussed in Giroletti et al. (in preparation). The source is reported in Table 1, but has not been considered in the statistical considerations and will not be considered in the future.

We refer to the "arcsecond core" for the measure of the nuclear emission at arcsecond resolution provided by VLA observations at $5 \mathrm{GHz}$ (see e.g. Giovannini et al. 1988), while we use the term "VLBI core" to refer to the unresolved compact component visible in the VLBI images at milliarcsecond resolution.

\section{Observations and data reduction}

\subsection{VLBA observations at $5 \mathrm{GHz}$}

We obtained Very Long Baseline Array (VLBA) observing time at $5 \mathrm{GHz}$ to produce high-resolution images for 26 sources of

1 The National Radio Astronomy Observatory is operated by Associated Universities, Inc., under cooperative agreement with the National Science Foundation. the BCS. For these observations, we selected sources with an estimated nuclear flux density $S_{\mathrm{c}}(5.0)$ at arcsecond resolution $>5 \mathrm{mJy}$.

Because in most cases the VLBA target source is not sufficiently strong for fringe-fitting and self-calibration, we used the phase referencing technique. Moreover, calibration of atmospheric effects for either imaging or astrometric experiments can be improved by the use of multiple phase calibrators that enable multi-parameter solutions for phase effects in the atmosphere. We therefore adopted a multi-calibrator phase calibration. In addition, a relatively long integration time for each source has been used to obtain good $(u, v)$-coverage, necessary to properly map complex faint structures.

Observations were made with the full VLBA. Each source was observed with short scans at different hour angles to ensure good $(u, v)$-coverage. We observed in full polarization (RCP and LCP) with 4 IFs (central frequency $4.971 \mathrm{GHz}, 4.979 \mathrm{GHz}$, $4.987 \mathrm{GHz}$ and $4.995 \mathrm{GHz}$ ). Both right- and left-circular polarizations were recorded using 2 bit sampling across a bandwidth of $8 \mathrm{MHz}$. The VLBA correlator produced 16 frequency channels per IF/polarisation for a total aggregate bit rate of $256 \mathrm{Mbs}$. In order to improve the VLBI astrometric accuracy and image quality of a target source, we observed more than one reference calibrator along with the target. We scheduled the observation so as to use the AIPS task ATMCA, which combines the phase or multi-band delay information from several calibrators. Each pointing on a target source was bracketed by a phase calibrator scan using a 4 min duty cycle $(2.5 \mathrm{~min}$ on source, $1.5 \mathrm{~min}$ on phase calibrator). After 4 scans of target and phase calibrators we introduced one ATMCA calibrator scan (1.5 min on each ATMCA calibrator). The total observing time on each target is about $110 \mathrm{~min}$. Calibrators were chosen from the VLBA calibrator list to be bright and close to the source; in Table 2, we report the list of the selected calibrators. Short scans on strong sources were interspersed with the targets and the calibrators as fringe finders. The observations were correlated in Socorro, NM. Postcorrelation processing used the NRAO AIPS package (Cotton 1993) and the Caltech Difmap packages (Pearson et al. 1994). We produced also images of the calibrators in order to obtain and apply more accurate phase and gain corrections to the multi-sources file during the initial calibration steps; then we determined the absolute position of the sources. We give the coordinates of the core candidate of each source in Table 2 . Initial images were derived using phase-referencing, then self-calibrated depending on SNR. The signal-to-noise allowed for phase selfcalibration on a very few sources.

Final images were obtained using the AIPS and Difmap packages. We produce images with natural and uniform weights. The important parameters for the final images of the observed sources are summarized in Table 3. The VLBI core properties have been estimated by using Modelfit in Difmap. The total VLBI flux is the sum of all fitted components. The noise level was estimated from the final images.

\subsection{VLBA observations at $1.6 \mathrm{GHz}$}

Observations of 10 objects were also obtained in phase reference mode with the VLBA on 2003 August 07 and 2003 August 30 as part of projects BG136A, and B. Observations were performed in full polarization (RCP and LCP) with two IFs (central frequencies 1659.49 MHz and 1667.49 MHz). Both right- and left-circular polarizations were recorded using 2 bit sampling across a bandwidth of $8 \mathrm{MHz}$. The VLBA correlator produced 
16 frequency channels per IF/polarisation for a total aggregate bit rate of $128 \mathrm{Mbs}$.

Each pointing on a target source was bracketed by a calibrator scan in a $5 \mathrm{~min}$. duty cycle $(3 \mathrm{~min}$. on source, $2 \mathrm{~min}$. on the calibrator). Two groups of (typically) 11 duty cycles were executed for each source at different hour angles, resulting in a total of $\sim 66$ min per target, with a good coverage of the $(u, v)$-plane. Calibrators were chosen from the VLBA Calibrators List to be bright and close to the source; a list of sources with their relative calibrator is reported together with the $5 \mathrm{GHz}$ calibrators used in Table 2.

The correlation was performed in Socorro and the initial calibrations were done within AIPS. Global fringe fitting was performed on all calibrators and the solutions were applied to the targets using a two-point interpolation. We then produced images of the calibrators in order to obtain and apply more accurate phase and gain corrections to the sources; we also determined from preliminary maps the absolute position of the sources, which we used thereafter. The final calibrated single-source datasets were exported to Difmap, for imaging and self-calibration. We produced images with the same procedure described for the $5 \mathrm{GHz}$ data, and the relevant parameters are summarized in Table 3.

\section{Results}

We present here new observations for 33 sources (including the spiral galaxy $0722+30$, see above). We detected all sources except $0722+30$ and $1339+26$ at $5 \mathrm{GHz}$, and $1448+63$ (3C 305$)$ at $1.6 \mathrm{GHz}$. Among detected sources a few exhibit an extended structure that we will discuss in detail below. For all the other sources we give a short note and for all resolved sources we present an image. A summary of results are reported in Table 3.

\subsection{Sources of special interest}

\subsection{1. $0836+29-I$ (4C 29.30)}

This source $(z=0.0647$, also referred to as B2 0836+29A) has been often confused in the literature with the $\mathrm{cD}$ galaxy B2 0836+29 $(0836+29-$ II, at $z=0.079)$, the brightest galaxy in Abell 690, which was studied by Venturi et al. (1995).

0836+29-I was studied in detail by van Breugel et al. (1986), and recently by Jamrozy et al. (2007). It is a possible merger system (van Breugel et al. 1986). On the large scale the source shows clear evidence of intermittent radio activity. There is a large scale structure about $9^{\prime}$ in size, with an estimated age of about 200 Myr (Jamrozy et al. 2007); a more compact structure shows a central core and two bright spots and an extended emission about $1^{\prime}$ in size with an age lower than 100 Myr. The inferred spectral age for the inner double is $33 \mathrm{Myr}$ (Jamrozy et al. 2007). At higher resolution (van Breugel et al. 1986) the core source (component $\mathrm{C} 2$ ) is visible along with a bright one-sided jet about $3^{\prime \prime}$ in size, which terminates with a bright spot $(\mathrm{C} 1)$.

In our low resolution VLBA image (Fig. 1), the parsec scale structure looks like a scaled version of the arcsecond scale structure shown by van Breugel et al. (1986). At higher resolution (Fig. 2) the Northern component is resolved into a core-jet structure, and the Southern component is resolved into a bright knot in the most external region. Although we lack spectral information, we can identify the nuclear source as the Northern parsec scale component, since it is the only unresolved structure by a Gaussian fit and because of homogeneity with the arcsecond scale. From the jet to counter-jet ratio $(>50)$ we estimate that the

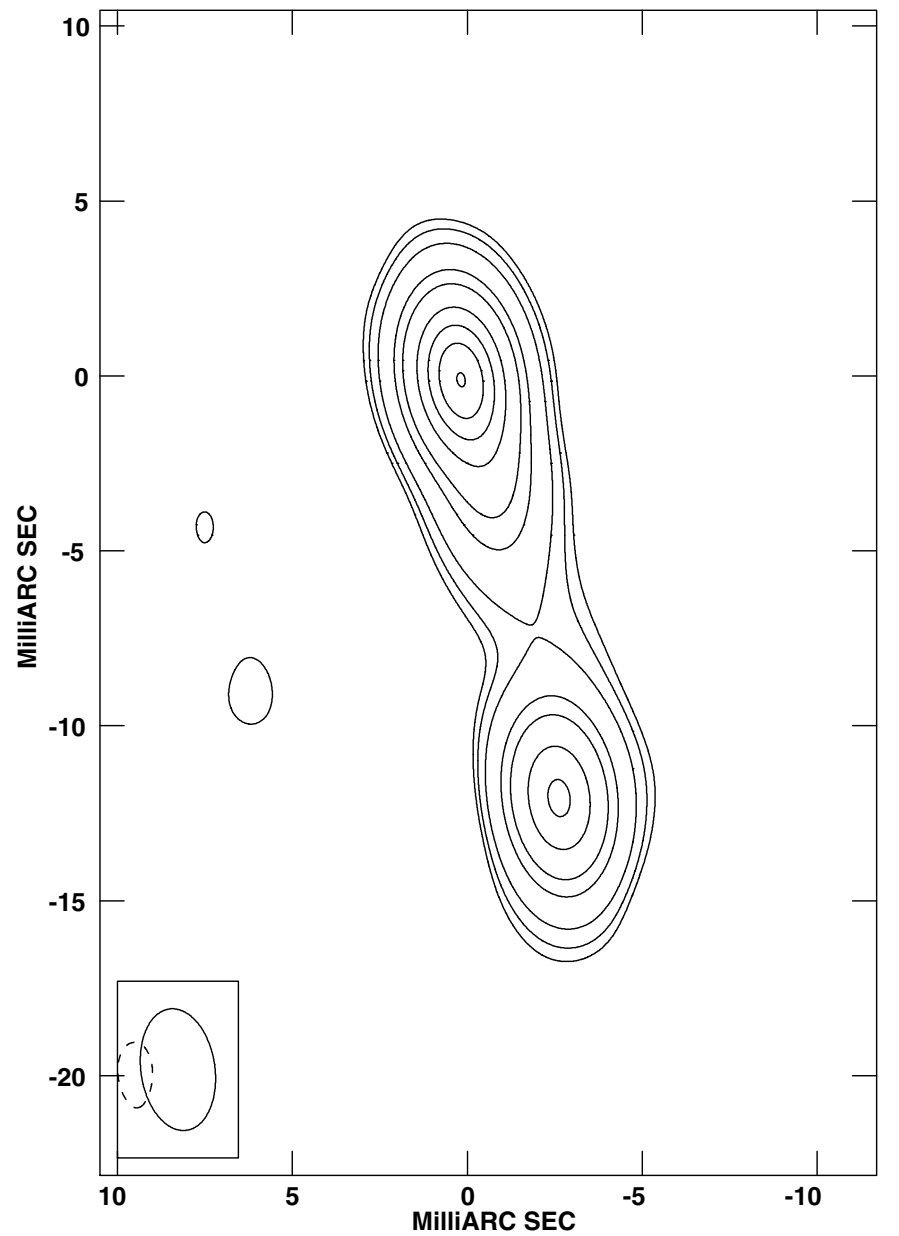

Fig. 1. VLBA image at $5 \mathrm{GHz}$ of $0836+29-\mathrm{I}$ at low resolution $\left(\mathrm{HPBW}=35 \times 21\right.$ mas in PA $8^{\circ}$ ). The noise level is $0.1 \mathrm{mJy} / \mathrm{beam}$. Levels are $-0.30 .30 .513510152025 \mathrm{mJy} / \mathrm{beam}$.

orientation angle has to be $\theta<49^{\circ}$ and the jet velocity $\beta>0.65$. Assuming a high jet velocity with a Lorentz factor in the range 3 to 10 (Giovannini et al. 2001) and an orientation angle $\theta \sim 40^{\circ}$, we can derive the intrinsic jet length and age of single components. In this scenario the first knot after the core (Fig. 2), is $\sim 15$ yr old and the Southern knot is $\sim 70$ yr old.

The structure of this source is in agreement with a quasiperiodic activity visible in the small as well as on the large scale. Assuming the same orientation angle and velocity of the jet on the arcsecond scale $\sim 0.6 \mathrm{c}$ (in agreement with the jet sidedness) we can estimate an age $\sim 10^{4}$ yr for the one-sided jet structure present in van Breugel et al. (1986). We note that Jamrozy et al. (2007) discussed the evidence of a strong outburst in between 1990 and 2005, which could be related to the presence of the new VLBI component with an estimated age of $15 \mathrm{yr}$. In Tables 1 and 4 we report the more recent arcsecond core flux density from Jamrozy et al. (2007). It is important to note that during the long time range of the activity of this source with many periods of restarting activity the jet position angle was constant in time.

\subsection{2. $1350+31(3 C 293)$}

This peculiar source has been studied in detail in the radio and optical bands. Recent results are presented in Beswick et al. (2004) and Floyd et al. (2006), where the source structure is discussed from the sub-arcsec to the arcminute scale. In 


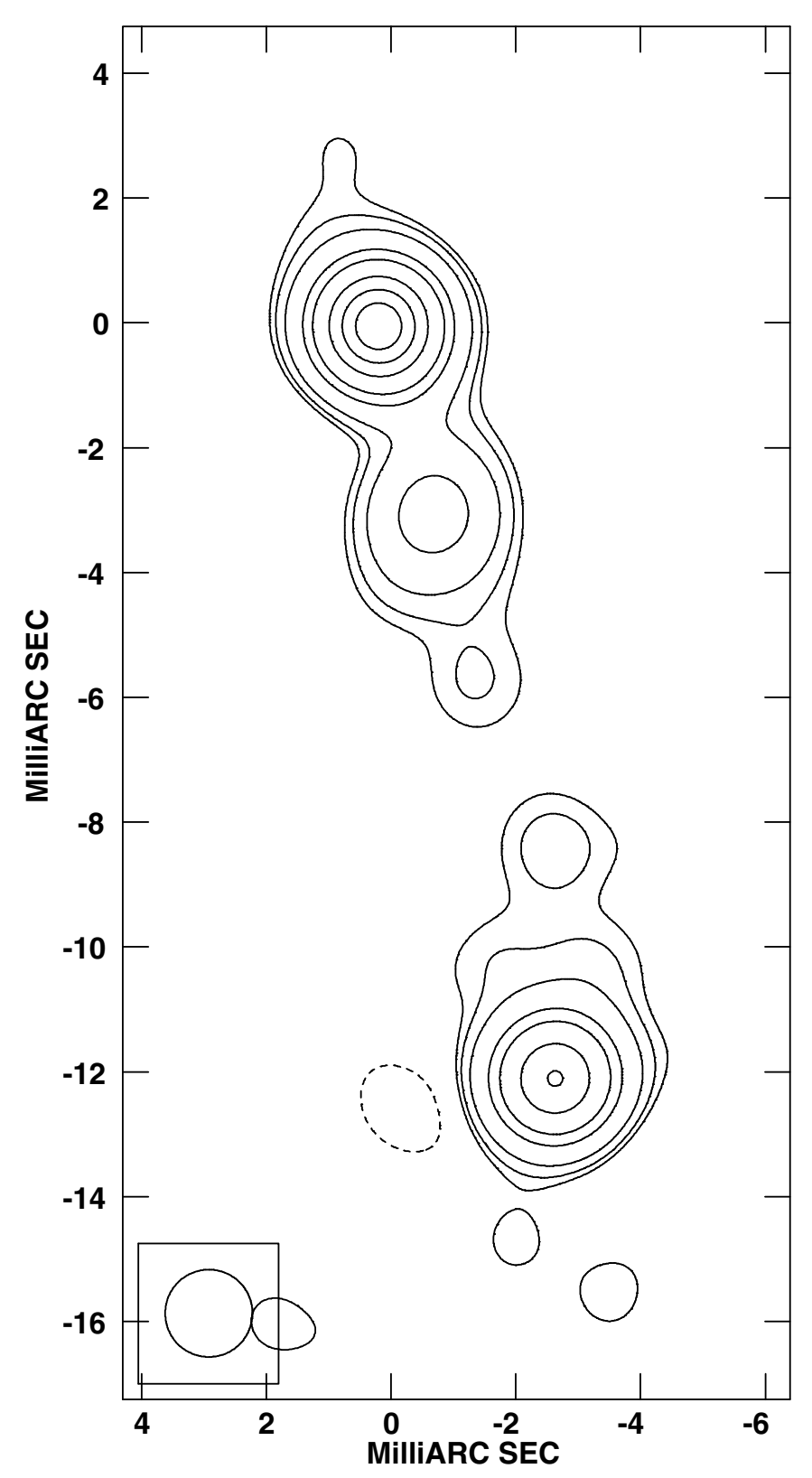

Fig. 2. VLBA image at $5 \mathrm{GHz}$ of $0836+29-\mathrm{I}$ at high resolution ( $\mathrm{HPBW}=1.4 \mathrm{mas}$ ). The noise level is $0.08 \mathrm{mJy} /$ beam and contours are: $-0.30 .30 .515101520 \mathrm{mJy} /$ beam. The nuclear source has been identified with the Northern compact component (see text).

Giovannini et al. (2005), we presented a VLBI image at $5 \mathrm{GHz}$ where a possible symmetric two-sided jet structure is present at mas resolution.

Because of the complex nature of the inner structure, we reobserved the source with the VLBA at $1.6 \mathrm{GHz}$ in phase reference mode. We show the new $1.6 \mathrm{GHz}$ image of the core in Fig. 3. In agreement with the $5 \mathrm{GHz}$ VLBA image, we have a two-sided structure with symmetric jets. The jet emission is detected on both sides up to 20 mas from the core, with the eastern jet being slightly brighter. Hereafter, we refer to it as the main jet(J), and to the western one as the counterjet (CJ). This is also in agreement with the indication discussed by Beswick et al. (2004), although their suggested jet orientation and velocity are in contrast with the high symmetry of the VLBA jet. From visibility model fitting, we derive total flux densities for the core

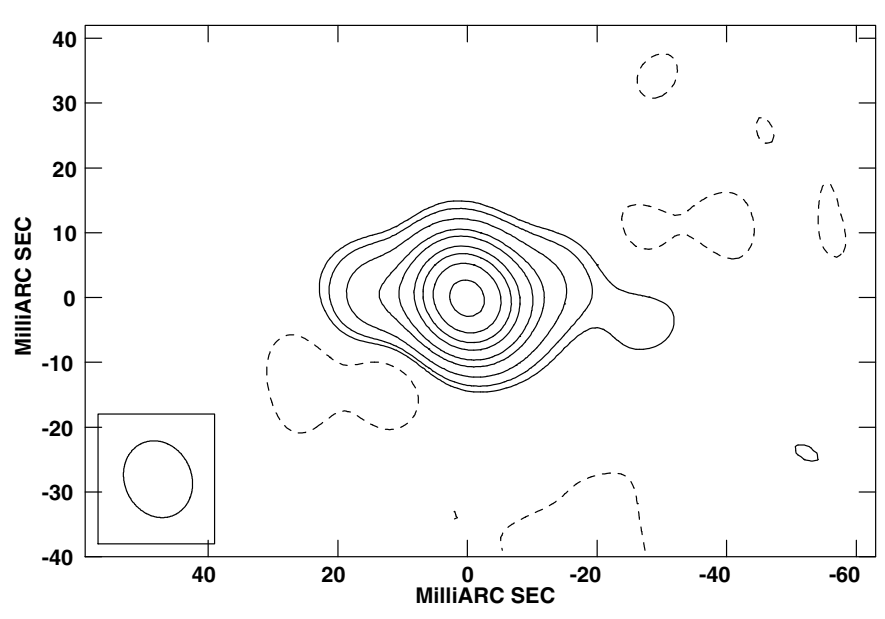

Fig. 3. 1.6 GHz VLBA image of $1350+31$ (3C 293). The HPBW is $12 \times$ 10 mas in PA $24^{\circ}$. The noise is $0.1 \mathrm{mJy} /$ beam, contours are -0.30 .30 .5$ $123571015 \mathrm{mJy} /$ beam.

and the jets of $S_{\mathrm{c}}=17 \mathrm{mJy}, S_{j}=2.5 \mathrm{mJy}$, and $S_{\mathrm{cj}}=2.0 \mathrm{mJy}$. The core spectral index is $\alpha_{1.6}^{5}=0.05$.

On the shortest baselines, we clearly detect a significant amount of extended flux $(S \sim 200 \mathrm{mJy})$ and we were able to image the MERLIN knots E1, E2, and E3 (see Fig. 3 in Beswick et al. 2004), while components on the west side are not revealed (data are reported in Table 3 ). We then reduced archival VLA data in order to better study the connection between the subarcsecond and the arcsecond structure. In Fig. 4, we show a $5 \mathrm{GHz}$ sub-arcsecond VLA+PT image. This reveals the inner two-sided jet structure with a fainter diffuse emission. On even larger scales (Fig. 5), VLA 1.4 GHz data reveal more extended, fainter emission along a different PA.

From a comparison between different images we infer that the sub-arcsecond structure in the E-W direction is related to restarted activity of the central AGN. The large change in PA with respect to the extended lobes, however, is not due to a PA change from the restarted nuclear activity but appears constant in time and is most likely produced by the jet interaction with a rotating disk as discussed in van Breugel \& Fomalont (1984). In this scenario we expect that the jet at $\sim 2.5 \mathrm{kpc}$ from the core is no longer relativistic.

\subsection{3. $1502+26(3 \mathrm{C} 310)$}

This source is identified with a magnitude 15 elliptical galaxy at $z=0.0538$ having clearly extended X-ray emission, first detected with the Einstein satellite by Burns et al. (1981). In the radio band at low resolution, it appears as a relaxed double van Breugel \& Fomalont (1984) with a steep radio spectrum $\left(\alpha_{750}^{26}=0.9 ; \alpha_{10.7}^{0.75}=1.4\right)$. The low brightness lobes exhibit a complex structure with filaments and bubbles. There is an unresolved radio core with a faint extension from the core to the North, extended $5^{\prime \prime}$, possibly identified as a weak jet (van Breugel \& Fomalont 1984).

The parsec scale images are complex. In Giovannini et al. (2005), we showed a structure interpreted as one-sided emission, in contrast with an image by Gizani et al. (2002). Because of this discrepancy and the short exposure and low flux density, we reobserved this source at 5 and $1.6 \mathrm{GHz}$ in phase reference mode. In Fig. 6 we show a $5 \mathrm{GHz}$ image at full resolution, where an extended complex structure oriented in the E-W direction, almost perpendicular to the kiloparsec scale structure is present. The 


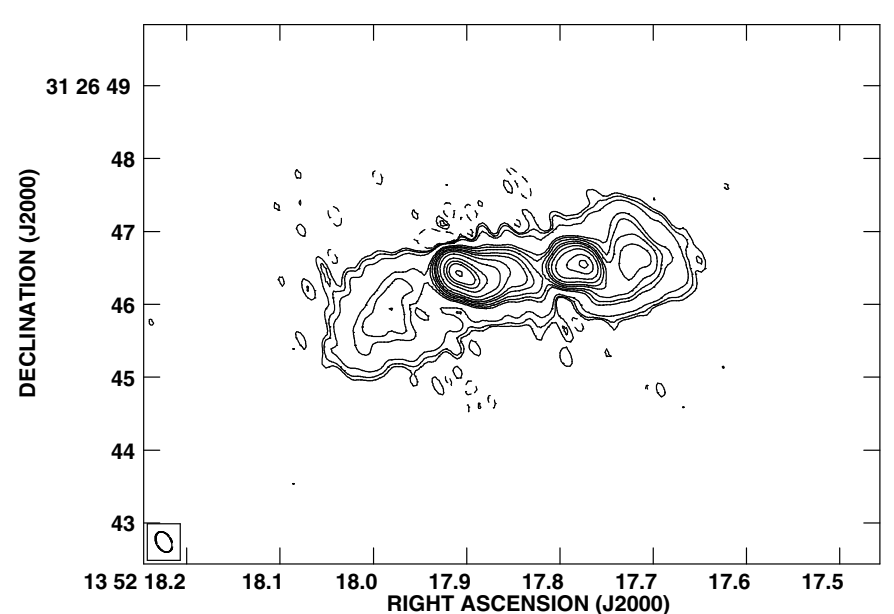

Fig. 4. $5 \mathrm{GHz}$ image of $1350+31$ (3C 293) obtained with VLA+PT. The HPBW is $0.3^{\prime \prime} \times 0.2^{\prime \prime}$ in PA $30^{\circ}$. The noise level is $0.06 \mathrm{mJy} / \mathrm{beam}$ and levels are: $-0.30 .30 .51571015203050100150200300 \mathrm{mJy} /$ beam.

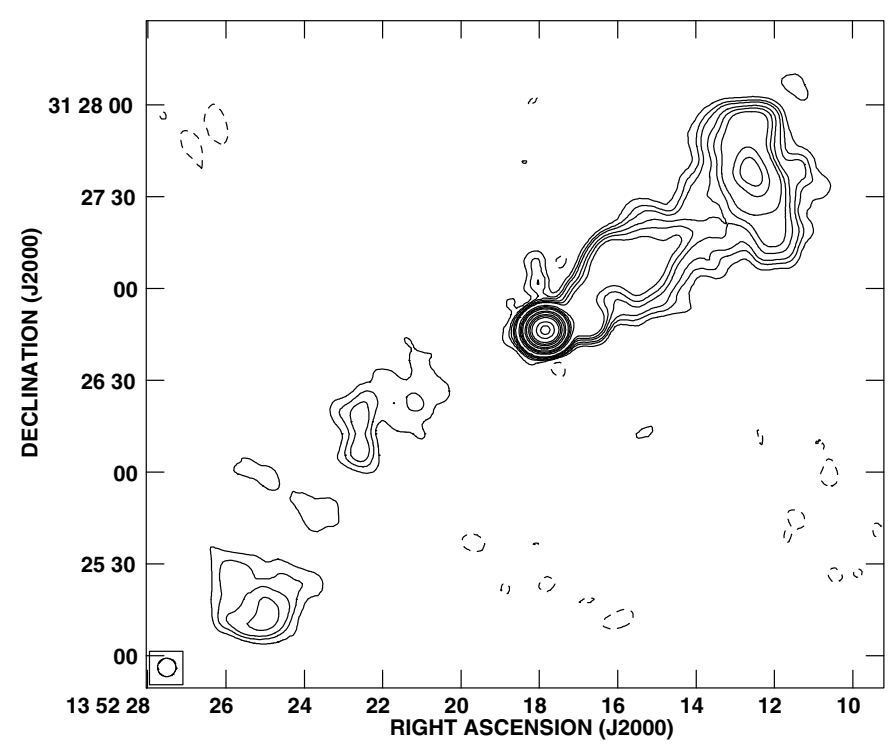

Fig. 5. VLA image at $1.4 \mathrm{GHz}$ of $3 \mathrm{C} 293$. The HPBW is $6 "$; the noise level is $0.26 \mathrm{mJy} / \mathrm{beam}$, and levels are: -11235710305070100200 300500700100020003000 mJy/beam.

symmetry of this structure suggests the presence of a nuclear central emission with two almost symmetrical lobes connected by a bridge (jets?). At lower resolution (Fig. 7) the double EW structure is confirmed, moreover some emission on the top (North) of the brightest lobe is visible. At $1.6 \mathrm{GHz}$ (Fig. 8) the source presents two main components along the East-West direction plus a low brightness emission extending to the North, in agreement with the low resolution $5 \mathrm{GHz}$ image, and with Gizani et al. (2002). In all images the source brightness is very low, at the mJy level at $5 \mathrm{GHz}$ and with a peak flux density of $8.5 \mathrm{mJy}$ at $1.6 \mathrm{GHz}$.

In order to discuss the nature of the components, we produced a map of the spectral index (defined as $S_{v} \propto v^{-\alpha}$ ) distribution, using images at 5 and $1.6 \mathrm{GHz}$ with the same angular resolution. The spectral index is inverted in the central region of the E-W structure $(-0.3)$ and steep in the two symmetric lobes (on the average 0.9 at $\mathrm{E}$ and 1.1 at $\mathrm{W}$ ). Despite the low signal to noise ratio we are able also to estimate the spectral index in the North region which is still steep $(\sim 1.3)$, albeit with a large uncertainty

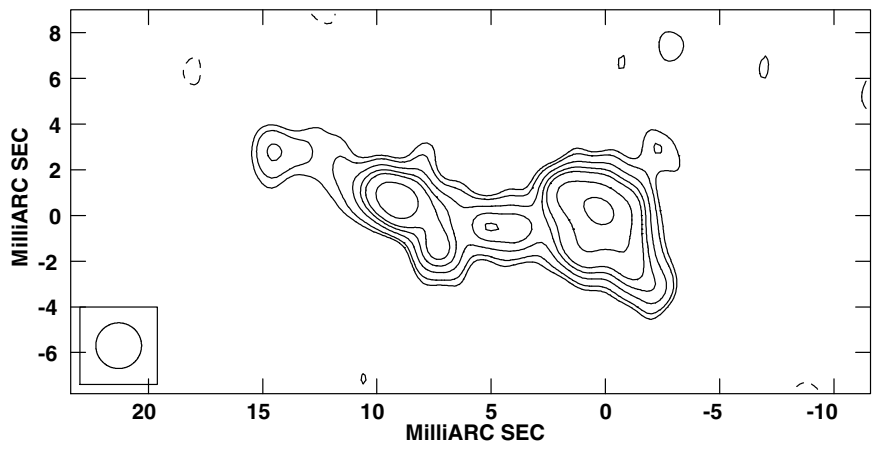

Fig. 6. VLBA image of $1502+26(3 \mathrm{C} 310)$ at $5 \mathrm{GHz}$. The HPBW is 2 mas. The noise level is $0.06 \mathrm{mJy} / \mathrm{beam}$ and levels are: -0.20 .150 .2$ $0.30 .40 .50 .71 \mathrm{mJy} / \mathrm{beam}$.

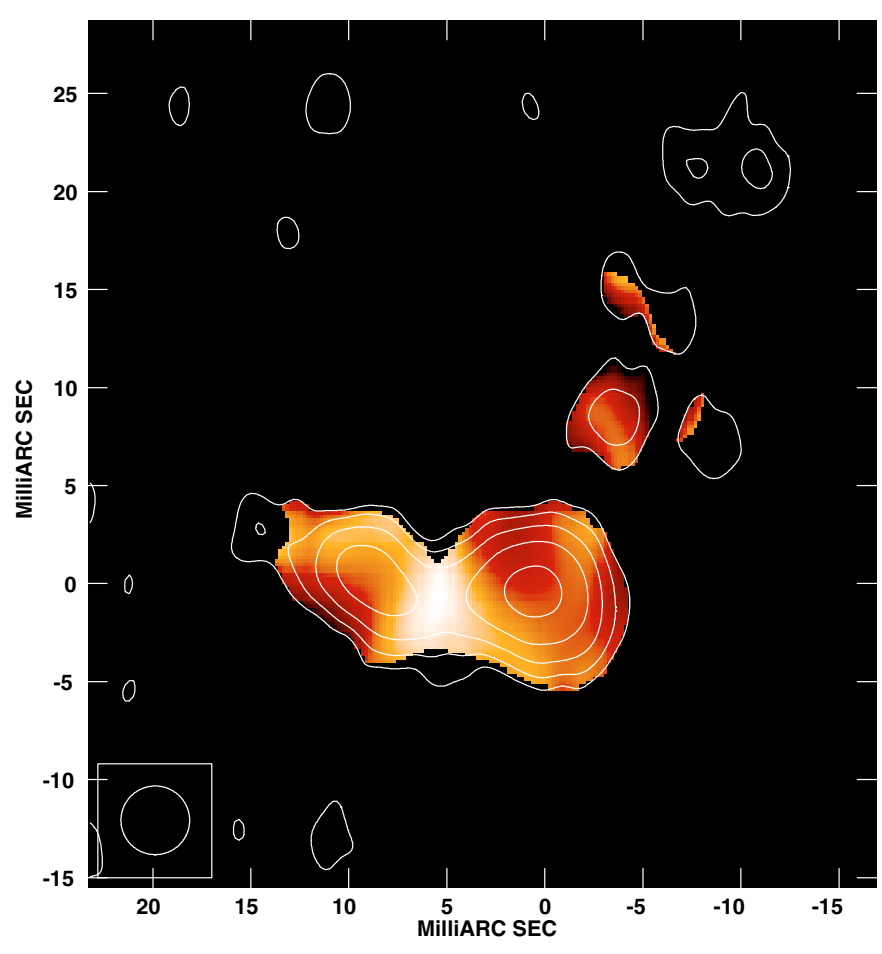

Fig. 7. VLBA image of $1502+26(3 \mathrm{C} 310)$ at $5 \mathrm{GHz}$ with a HPBW $=3.5$ mas superimposed to the spectral index image (colour) between 1.6 and $5 \mathrm{GHz}$. The range in the colour image is from -0.3 (white) to 2.0 (dark red). The noise level in the contour image is $0.04 \mathrm{mJy} / \mathrm{beam}$ and levels are: $0.10 .20 .40 .81 .6 \mathrm{mJy} / \mathrm{beam}$.

(see Fig. 7). Therefore we confirm the identification of the core with the faint emission at the center of the parsec scale structure. The morphology is quite peculiar: we have a symmetric twosided emission in the E-W direction, i.e. almost perpendicular to the kiloparsec-scale emission. After about five milliarcseconds we have a symmetric change in the direction of the radio emission of about $80^{\circ}$ which then becomes aligned with the kiloparsec scale axis. A possible explanation for this Z-shaped structure so near to the core will be discussed in Sect. 5.3.

\subsection{Notes on other observed sources}

The remaining sources presented in this paper have simpler structures. They can be unresolved, have short jet-like features, or even remain undetected. Below we summarize the results on these sources, together with some information on the large-scale 


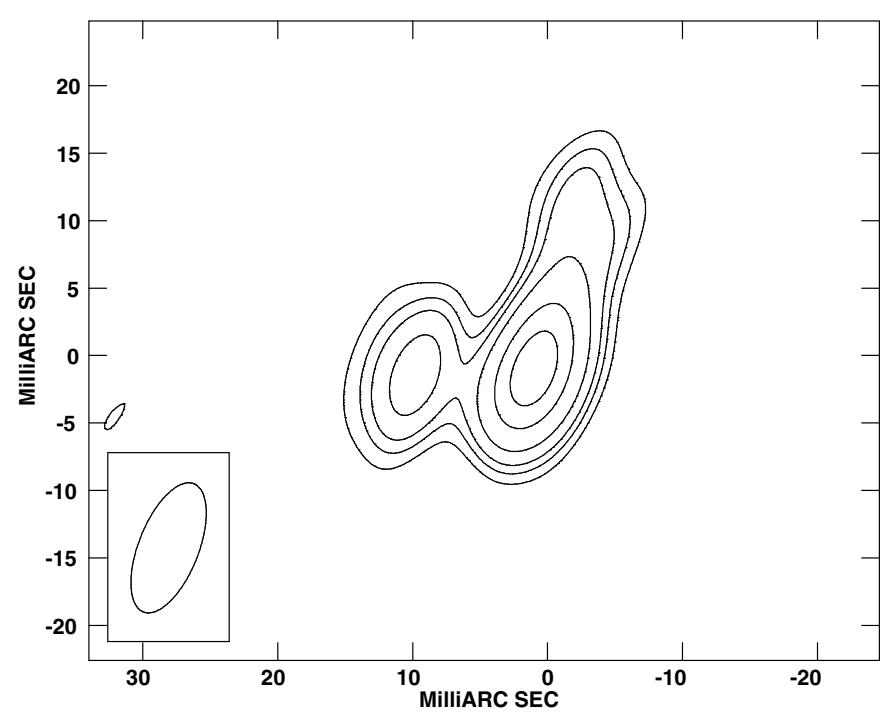

Fig. 8. VLBA image at $1.7 \mathrm{GHz}$ of $1502+26$ (3C 310), with a HPBW $=10.2 \times 4.5$ mas in PA $-22^{\circ}$. The noise is $0.2 \mathrm{mJy} / \mathrm{beam}$, and levels are: $-111.52357 \mathrm{mJy} /$ beam.

structure. We only skip 1346+26 (4C 26.42), a very peculiar radio source with a $\mathrm{Z}$ shaped morphology from sub-parsec to kiloparsec scales), since we will present a detailed discussion with more data in a separate paper (Liuzzo et al. 2009).

$0034+25$ (UGC367) - This radio galaxy, identified with an E galaxy at $z=0.032$, shows a wide angle tail morphology with an inner symmetric jet structure in $\mathrm{PA}=90^{\circ}$. It belongs to the Zwicky cluster 0034.4+2532. The host galaxy has an inner stellar disk perpendicular to the radio jet that has been interpreted as the signature of merging in the past (Gonzalez-Serrano et al. 1993). In our VLBA observation the source appears unresolved with total flux density $\sim 3.6 \mathrm{mJy}$. As the ratio $S_{\mathrm{VLBA}} / S_{\mathrm{c}}$ is only $36 \%$, most of the arcsecond flux is still missing on the milliarcsecond scale.

$0055+26$ (NGC 326) - This galaxy is the Brightest Cluster Galaxy (BCG) of the Zwicky cluster 0056.9+2636. It appears as a dumbbell galaxy with two equally bright nuclei with projected separation of $7^{\prime \prime}$. The large scale radio emission shows twin jets along direction PA $135^{\circ}$ (Murgia et al. 2001, and references therein). There is a peak in X-ray emission consistent with the location of the radio galaxy core. In our VLBA image (not shown here), we found nuclear emission with a possible slight extension in the same PA as the kiloparsec-scale jets, with a total flux density of $7.5 \mathrm{mJy}$.

$0149+35$ (NGC 708) - This galaxy is the BCG of the cooling cluster A262. At optical wavelengths, it is a low brightness galaxy whose nuclear regions are crossed by an irregular dust lane and patches of dust (Capetti et al. 2000). Feedback between the radio activity and the surrounding thermal gas is discussed by Blanton et al. (2004). In our VLBA image, this source appears unresolved with a total flux density $S_{5}=3.2 \mathrm{mJy}$. Some of the arcsecond core flux is lost on the parsec scale $\left(S_{\mathrm{VLBA}} / S_{\mathrm{c}} \sim 64 \%\right)$.

$0300+16(3 C 76.1)$ - On kiloparsec scales, 3C 76.1 is classified as an FR I radio galaxy; it was imaged at $6 \mathrm{~cm}$ by Vallèe (1982), and at $20 \mathrm{~cm}$ by Leahy \& Perley (1991). The source has twin jets with a faint $(\sim 8 \mathrm{mJy})$ radio core surrounded by an extended low brightness radio halo. In our VLBA image, we found an unresolved core with the total flux density $S_{5}=6.1 \mathrm{mJy}$.

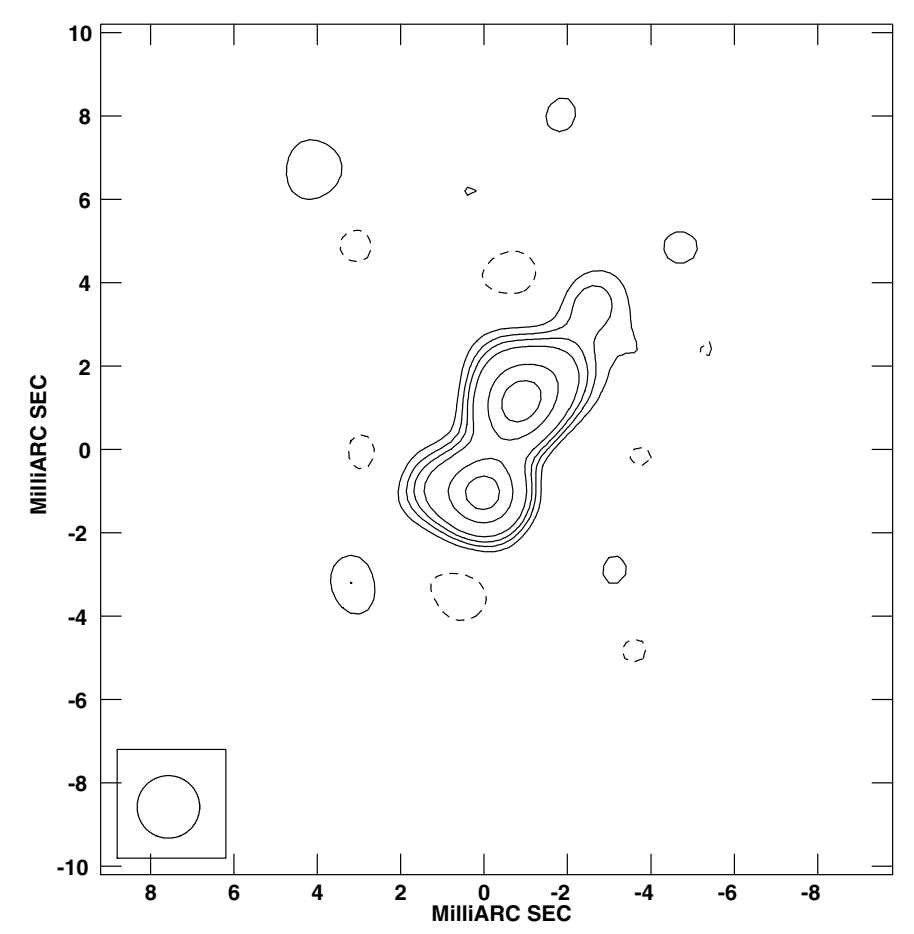

Fig. 9. VLBA image at $5 \mathrm{GHz}$ of $0708+32 \mathrm{~B}$. The HPBW is 1.5 mas, the noise level is $0.08 \mathrm{mJy} /$ beam and levels are: -0.30 .30 .50 .712$ $3 \mathrm{mJy} /$ beam.

Some arcsecond core flux is lost on the parsec scale $\left(S_{\mathrm{VLBA}} / S_{\mathrm{c}} \sim\right.$ $60 \%)$.

$0356+10-(3 C 98)-$ The host galaxy is a typical narrowline radio galaxy (NLRG), with extended emission lines studied by (Baum et al. 1988, 1990). On kiloparsec scales, 3C 98 shows a double-lobe FRII radio structure which spans 216 arcsec at $8.35 \mathrm{GHz}$, with a radio jet that crosses the northern lobe and terminates in a bright hotspot. There is little evidence of a southern jet, but a twin hotspot in the southern lobe is present (Hardcastle et al. 1998). The core of this symmetric FRII radio galaxy is pointlike in our VLBI image, with the total flux density $\sim 2.2$ mJy. Most of the arcsecond core flux is missing on the parsec scale $\left(S_{\mathrm{VLBA}} / S_{\mathrm{c}} \sim 24 \%\right)$, suggesting the presence of faint jets below our noise level and invisible because they are oriented in the plane of the sky.

$0708+32 \mathrm{~B}$. This source is classified as a low power compact source. In VLA images at $20 \mathrm{~cm}$ (Fanti et al. 1986) it shows a nuclear emission with two symmetric lobes $<10^{\prime \prime}(\sim 10 \mathrm{kpc})$ in size oriented in the N-S direction.

Higher resolution VLA data will be presented in Giroletti et al. (2009) to address the kiloparsec-scale structure of this source. In our VLBA images (Figs. 9 and 10), we detected a double structure oriented along $\mathrm{PA} \sim 150^{\circ}$ and extended $\sim 4$ mas. Since no spectral index information is available we are not able to identify the nuclear source, but the high degree of symmetry suggests an identification as a CSO with recurrent radio activity. The correlated VLBI flux density is $9.5 \mathrm{mJy}$ about $70 \%$ of the arcsecond core flux density.

$0722+30$ This radio source at $z=0.0188$ is the only one identified with a spiral galaxy (see Capetti et al. 2000). It is a highly inclined galaxy with a strong absorption associated with the disk and a bulge-like component. The radio emission is quite peculiar showing two symmetric jet-like features at an angle of about $45^{\circ}$ from the disk (see Fanti et al. 1986). New 


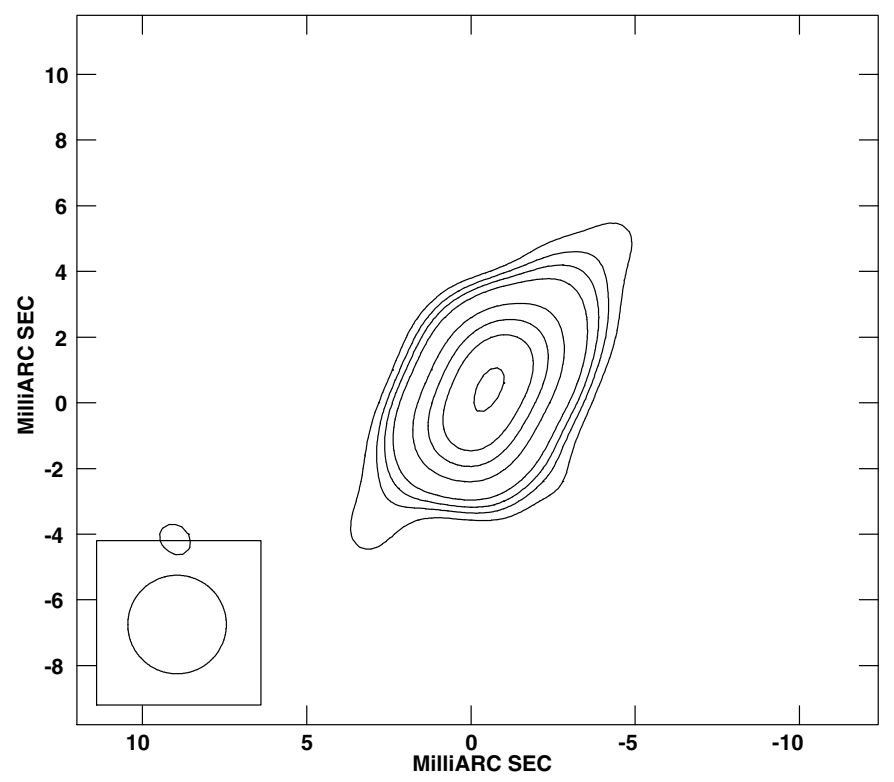

Fig. 10. VLBA image at $5 \mathrm{GHz}$ of $0708+32 \mathrm{~B}$. The HPBW is 3 mas, the noise level is $0.09 \mathrm{mJy} /$ beam and levels are: -0.30 .30 .50 .71234$ $5 \mathrm{mJy} /$ beam.

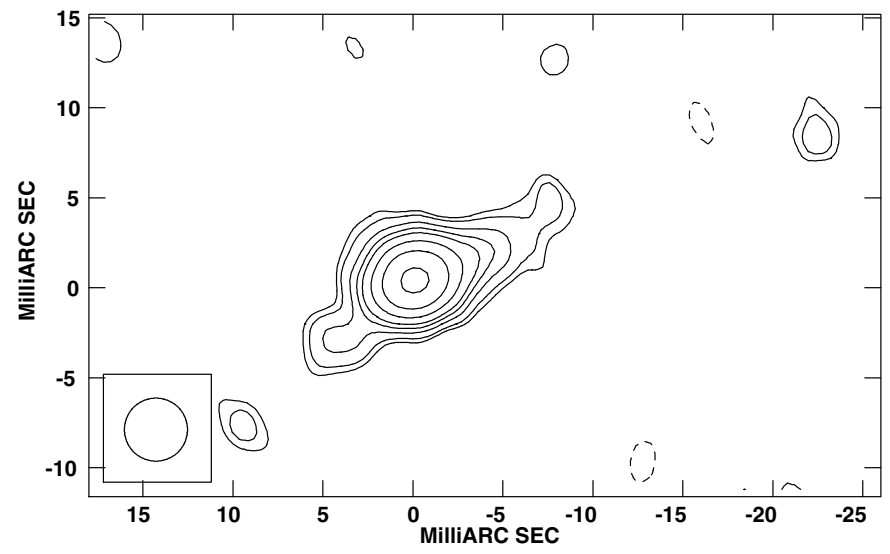

Fig. 11. The VLBA image at $5 \mathrm{GHz}$ of $0802+24$ (3C 192). Contour levs are $-0.20 .150 .20 .30 .40 .50 .711 .5 \mathrm{mJy}^{-1}$ beam $^{-1}$, and the HPBW is 3.5 mas. The noise level is $0.05 \mathrm{mJy} /$ beam.

high resolution VLA images will be discussed in Giroletti et al. (2009). The source is undetected in our VLBA image, with a $S_{\text {peak }}<0.3 \mathrm{mJy} /$ beam at $5 \mathrm{GHz}$. Because of the optical identification this source will not be considered in the following discussion.

0802+24 (3C 192) - On large scale, this radio source shows an " $\mathrm{X}$ " symmetric double-lobe structure which extends $\sim 200$ arcsec at $8.35 \mathrm{GHz}$, showing bright hotspots at the end of the lobes (Baum et al. 1988; Hardcastle et al. 1998).

Extended narrow line emission has been detected in this galaxy. In our VLBA image (Fig. 11), the source appears twosided, with jets at the same orientation as the kiloparsec-scale jets $\left(\mathrm{PA} \sim-80^{\circ}\right.$ ). The core flux density is $\sim 2.1 \mathrm{mJy}$ and the total flux density is $\sim 3.6 \mathrm{mJy}$. The correlated VLBI flux density is $\sim 50 \%$ of the arcsecond core flux.

$0838+32(4 C 32.26)$ - This radio source is associated with a dumbbell galaxy identified as the brightest galaxy in Abell 695, although recent works claim that it is at the centre of a group unrelated and much closer than the Abell cluster it was originally

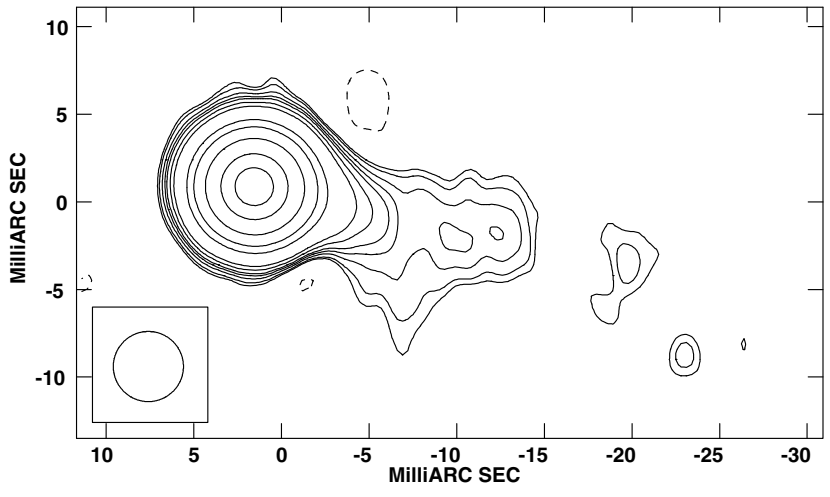

Fig. 12. VLBA image of $1113+29$ at $5 \mathrm{GHz}$. The HPBW is 4 mas, the noise level is $0.06 \mathrm{mJy} /$ beam and levels are: -0.20 .150 .20 .30 .40 .5$ $0.7135102030 \mathrm{mJy} /$ beam.

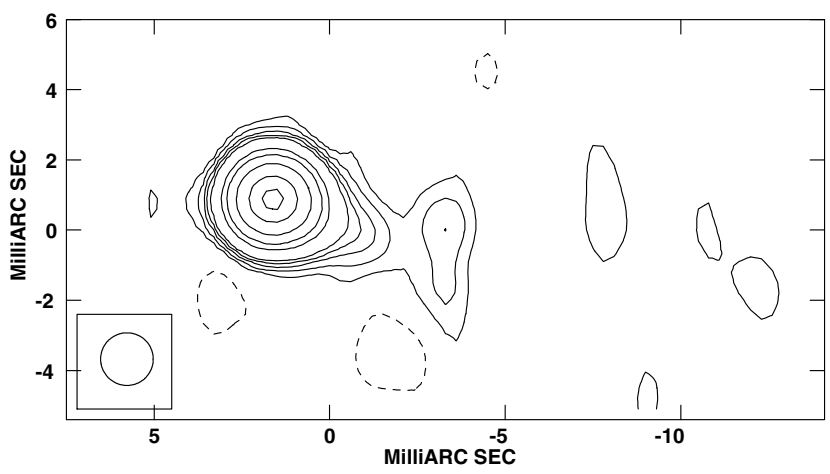

Fig. 13. VLBA image of $1113+29$ at $5 \mathrm{GHz}$. The HPBW is 1.5 mas, the noise level is $0.07 \mathrm{mJy} /$ beam, and levels are: -0.20 .150 .30 .50 .713$ $5102030 \mathrm{mJy} /$ beam.

identified with (Jetha et al. 2008). At low resolution (Valentijn 1979; Jetha et al. 2008) it appears to be a Wide Angle Tailed (WAT) source, but at high resolution it shows a compact FRII structure with core flux density $S_{\mathrm{c}, 1.4} \sim 7.5 \mathrm{mJy}$ (Machalski 1998). The radio structure suggests a possible restarting activity and X-ray data suggest that the currently active lobes are expanding supersonically (Jetha et al. 2008). In our VLBA image, the source is unresolved with total flux density $S_{5}=7.0 \mathrm{mJy}$, with little or no arcsecond core flux missing on parsec scales $\left(S_{\mathrm{VLBA}} / S_{\mathrm{c}} \sim 90 \%\right)$.

0915+32 - The VLA radio map by Fanti et al. (1987) shows a two-sided jet in $\mathrm{PA}=28^{\circ}$ with mirror symmetry. The radio structure could be due to gravitational interactions with a nearby spiral galaxy (Parma et al. 1985). In our VLBA image, the source appears unresolved with a total flux density of $S_{5} \sim 13.6 \mathrm{mJy}$. No arcsecond core flux is lost on the mas scale $\left(S_{\mathrm{VLBA}} / S_{\mathrm{c}} \sim\right.$ $100 \%)$.

$1113+29(4 C 29.41)$ - This radio source is associated with the BCG of Abell 1213, which is part of a double system of galaxies close to the center of the cluster (Trussoni et al. 1997). At radio frequencies, it shows a double structure with linear size $\sim 61 \mathrm{kpc}$ and a total radio power at $1.4 \mathrm{GHz} \log P_{1.4}=24.7$ (Fanti et al. 1986). An extended weak X-ray emission has been found to be associated with the cluster (Ledlow et al. 2003).

In our VLBA image, the source shows an extended one-sided jet (Fig. 12), resolved in the high resolution image and suggestive of a limb-brightened structure (Fig. 13). The parsec-scale jet is on the same side as the main kiloparsec scale jet. The total 


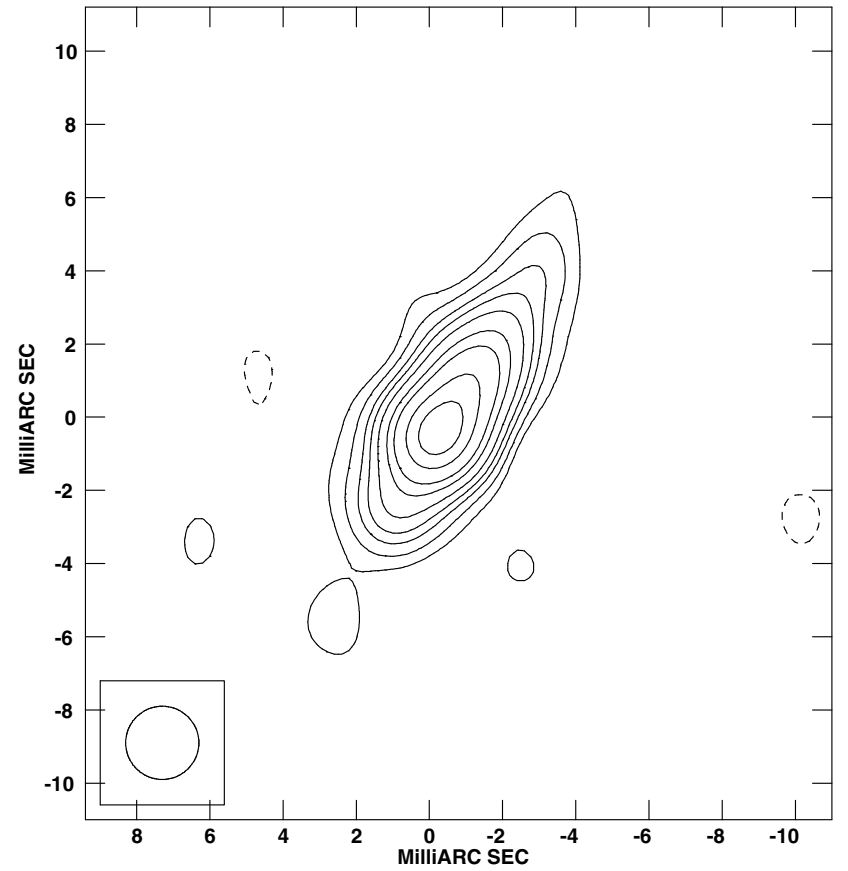

Fig. 14. VLBA image of $1251+27 \mathrm{~B}$ (3C277.3). The HPBW is 2 mas, the noise is $0.06 \mathrm{mJy} / \mathrm{beam}$, and levels are: -0.20 .150 .30 .50 .711 .5$ $234 \mathrm{mJy} / \mathrm{beam}$.

correlated flux in the VLBA image is $\sim 40 \mathrm{mJy}$, and therefore no arcsecond core flux is missing on the parsec scale.

$1116+28-$ The galaxy associated with this radio source is a double system at a distance of $z=0.0667$. At arcsecond resolution (Fanti et al. 1987), the radio source shows a Narrow Angle Tail (NAT) structure with two symmetric jets. Giovannini et al. (2005) showed a slightly resolved parsec-scale structure with two-sided jets oriented in the same direction as the kpcscale jets. Because of the large fraction of missing flux in the VLBI image we reobserved this source at 5 and $1.6 \mathrm{GHz}$. We do not confirm the two-sided jets previously reported. We consider therefore this source to be unresolved with total flux densities $S_{1.6}=9.5 \mathrm{mJy}, S_{5}=11.5 \mathrm{mJy}$, and a slightly inverted spectral index $\alpha_{1.6}^{5}=-0.17$. Most of the arcsecond core flux is still missing on the milliarcsecond scale at both frequencies $\left(S_{\mathrm{VLBA}} / S_{\mathrm{c}} \sim 40 \%\right)$.

$1122+39$ (NGC 3665) - At arcsecond resolution, this appears to be a core plus twin-jet radio source (Parma et al. 1986). In our VLBA image, it shows a pointlike structure with a total flux density $S_{5}=8.8 \mathrm{mJy}$. No arcsecond core flux is missing on the parcsec-scale $\left(S_{\mathrm{VLBA}} / S_{\mathrm{c}} \sim 100 \%\right)$

$1204+24$ - In the VLA image, this source appears as a FRI with faint symmetric lobes and a bright core (Fanti et al. 1986). In our VLBA image, it appears unresolved with a total flux density $S_{5}=5.3 \mathrm{mJy}$, corresponding to a fraction of the VLA core $S_{\mathrm{VLBA}} / S_{\mathrm{c}} \sim 66 \%$.

$1251+27 B(3 C 277.3)$ - The radio and optical properties of 3C 277.3 are described in detail by van Breugel et al. (1985). The elliptical host galaxy of 3C 277.3 is very smooth and regular; on kiloparsec-scales, the radio source extends $<60 \mathrm{kpc}$ and it has two wide and diffuse lobes with a few brightness enhancements (southern jet and northern hot spot).

In our VLBA image (Fig. 14), this source shows a possible two-sided structure with total flux density $\sim 10.4 \mathrm{mJy}$ and a jet orientation quite similar to the kiloparsec-scale jet $\left(\triangle \mathrm{PA} \sim 20^{\circ}\right)$.

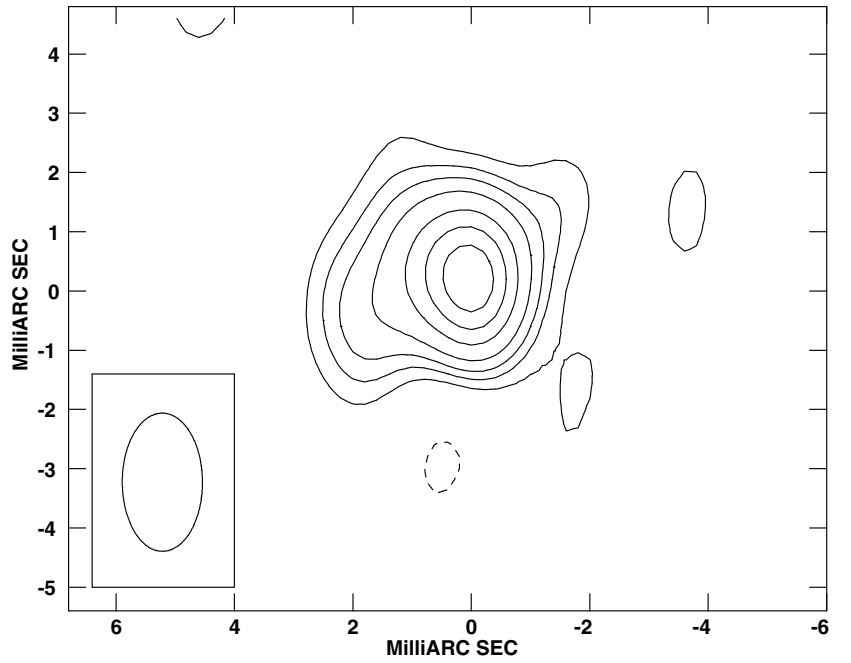

Fig. 15. VLBA image at $5 \mathrm{GHz}$ of $1319+42$ (3C 285). The HPBW is $2.3 \times 1.4$ mas in PA $0^{\circ}$. The noise level is $0.1 \mathrm{mJy} /$ beam and levels are: $-0.30 .30 .50 .711 .522 .5 \mathrm{mJy} / \mathrm{beam}$.

The core flux density is $\sim 6.2 \mathrm{mJy}$ and only $\sim 10 \%$ of the arcsecond flux is lost on the mas scale.

$1319+42(3 C 285)$ - The host galaxy of 3C 285 has been identified with the BCG of a group of galaxies (Sandage 1972). 3C 285 is a classical double-lobed radio galaxy of 190 arcsec total extension at $4.86 \mathrm{GHz}$, with two hotspots and an eastern ridge showing curvature roughly along the line to the optical companion (Leahy \& Williams 1984; Hardcastle et al. 1998). In our VLBA image, the source is slightly resolved at the same PA as the kiloparsec-scale jets (Fig. 15) with a symmetric structure. The total flux density is $\sim 4.7 \mathrm{mJy}$; very little arcsecond core flux is lost on the milliarcsecond-scale $\left(S_{\mathrm{VLBA}} / S_{\mathrm{c}} \sim 80 \%\right)$

$1339+26$ (UGC 08669) - This radio galaxy has a head-tail morphology. It is the easternmost of a double system identified with the dominant member of the cluster Abell 1775. A small dark nuclear band $\left(\mathrm{PA} \sim 0^{\circ}\right)$ characterizes this otherwise regular galaxy (Capetti et al. 2000). In our VLBA image, this source is not detected above $0.3 \mathrm{mJy}_{\text {beam }}{ }^{-1}$.

$1357+28$ - The host of this radio source is a round elliptical galaxy with a small dust lane $\left(\mathrm{PA} \sim 90^{\circ}\right)$ bisecting the nuclear region (Capetti et al. 2000). The radio map by Fanti et al. (1987) shows a two-sided inner jet in PA $0.5^{\circ}$ that decollimates and bends at 30 arcsec from the core. Our VLBA image (Fig. 16) shows a slightly extended core suggesting a two-sided structure in PA $\sim 20^{\circ}$. The VLBA core flux density is $5.6 \mathrm{mJy}$ and some of the arcsecond core flux is missing on the milliarcsecond scale $\left(S_{\mathrm{VLBA}} / S_{\mathrm{c}} \sim 60\right)$.

$1422+26$ - An extended FR I galaxy, with two symmetric jets oriented east-west at arcsecond resolution. At $5 \mathrm{GHz}$, Giovannini et al. (2005) detect a weak (3 mJy) milliarcsecond scale source. The $1.6 \mathrm{GHz}$ observations presented in this work detect a larger flux density $\left(S_{1.6} \sim 9.3 \mathrm{mJy}\right)$, distributed in a misaligned and distorted structure (see Fig. 17).

We tentatively identify the peak in the $1.6 \mathrm{GHz}$ image with the nuclear source detected also at $5 \mathrm{GHz}$. In this scenario the spectral index is $\sim 0$ and the extended $1.6 \mathrm{GHz}$ structure is a onesided, distorted jet. More data are necessary to understand the nature of this source.

$1448+63$ (3C 305) - A well known double source, recently studied in detail by Massaro et al. (2009), it was only marginally detected in previous VLBA $5 \mathrm{GHz}$ observations 


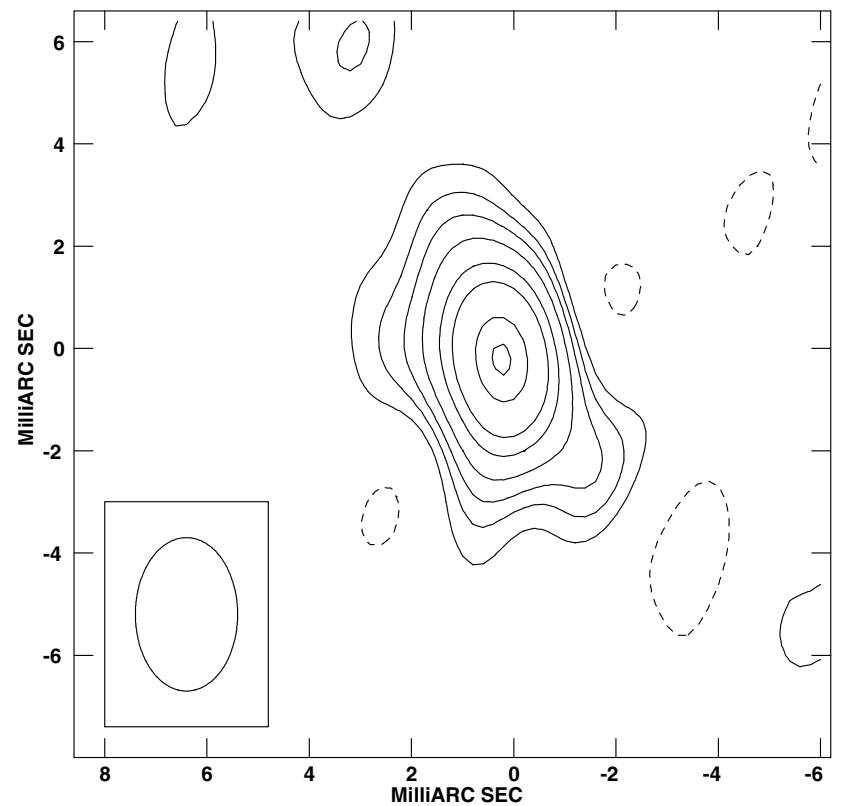

Fig. 16. VLBA image at $5 \mathrm{GHz}$ of $1357+28$. The HPBW is $3 \times 2$ mas in PA $0^{\circ}$. The noise is $0.15 \mathrm{mJy} / \mathrm{beam}$, and levels are: -0.30 .30 .50 .7$ $11.5233 .5 \mathrm{mJy} / \mathrm{beam}$.

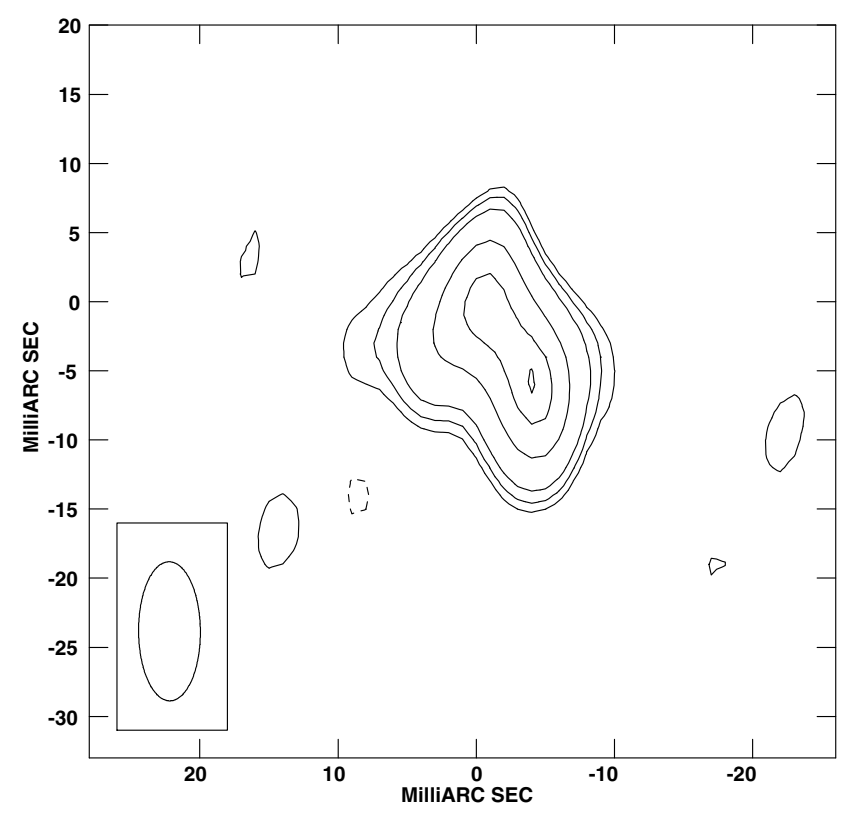

Fig. 17. VLBA image of $1422+26$ at $1.6 \mathrm{GHz}$. The HPBW is $10.1 \times$ $4.5 \mathrm{mas}$ in PA $0^{\circ}$. The noise is $0.15 \mathrm{mJy} / \mathrm{beam}$ and levels are: -0.50 .7$ $0.71233 .5 \mathrm{mJy} / \mathrm{beam}$.

(Giovannini et al. 2005). In our new observations at $1.6 \mathrm{GHz}$ the nuclear source is not detected, but there is a clear detection on the shortest baselines. The visibilities are well described by a two component model, separated by $\sim 1.5^{\prime \prime}$. These are probably the two hot spots visible in the MERLIN images (see Massaro et al. 2009).

$1529+24(3$ C 321) - An extended FR II narrow line radio galaxy, with optical evidence of double nuclei. The two components are clearly merging galaxies with evidence for a merger-triggered starburst activity (Roche \& Eales 2000). The kiloparsec-scale nuclear emission has been discussed by Baum et al. (1988).

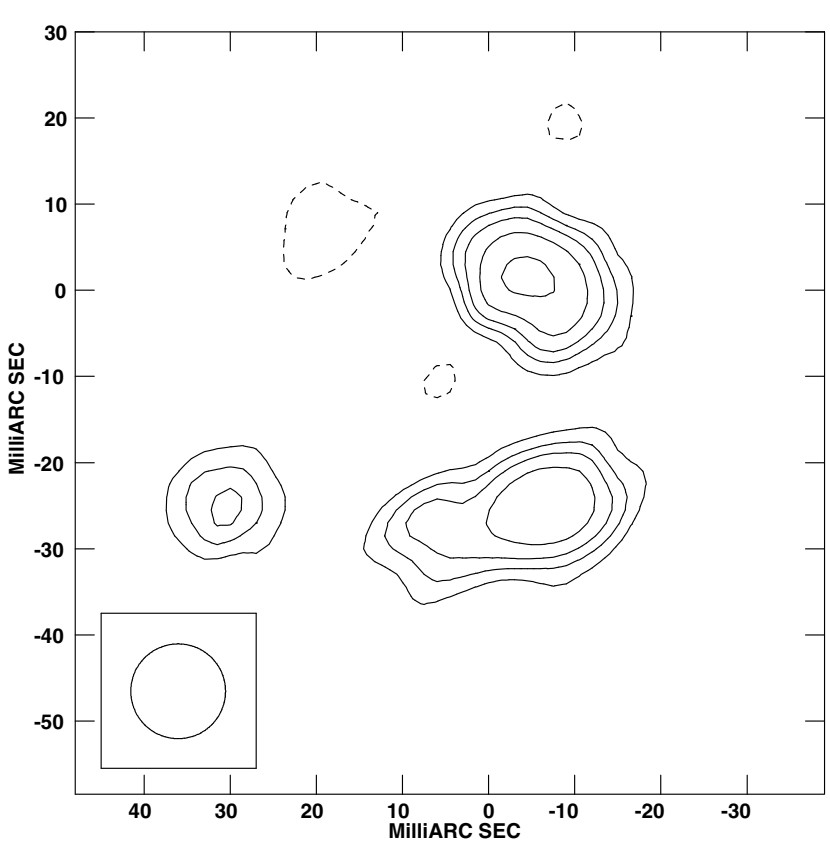

Fig. 18. VLBA image of $1529+24$ at $1.6 \mathrm{GHz}$. The HPBW is 11.0 mas. The noise is $0.1 \mathrm{mJy} /$ beam and levels are: $-0.3,0.30 .50 .7 \quad 1$ $1.5 \mathrm{mJy} / \mathrm{beam}$.

With the VLBA we detect at $1.6 \mathrm{GHz}$ some faint, diffuse emission, difficult to either clean or modelfit. A tentative visibility model fit reveals $\sim 7 \mathrm{mJy}$ at $1.6 \mathrm{GHz}$, in two nearly equal components separated by $0.03^{\prime \prime}$ (see Fig. 18).

A faint and complex structure is expected from the large difference between the point-like mas structure at $5 \mathrm{GHz}(\sim 3 \mathrm{mJy})$ and at $1.6 \mathrm{GHz}(\sim 7 \mathrm{mJy})$ and the arcsecond core flux density at $5 \mathrm{GHz}(\sim 30 \mathrm{mJy})$.

$1557+26-$ This is an unresolved source on arcsecond scale, with $S_{5}=30 \mathrm{mJy}$. Our $1.6 \mathrm{GHz}$ image recovers $\sim 10 \mathrm{mJy}$ in a relatively compact component. The spectral index with respect to the $5 \mathrm{GHz}$ data by Giovannini et al. (2005) is $\alpha_{1.6}^{5}=0.1$ in agreement with the core dominated one-sided structure found at $5 \mathrm{GHz}$. Visibility phases and amplitudes on the LA-PT baseline suggest that there could also be a more extended component, but it is not possible to constrain it with the present data.

1613+27 - A symmetric double on kiloparsec scales (Parma et al. 1986), with a $25 \mathrm{mJy}$ core at $5 \mathrm{GHz}$. Our 1.6 VLBA image reveals an unresolved core, with $S_{\mathrm{VLBA}, 1.6}=9.3 \mathrm{mJy}$, in agreement with VLBA at $5 \mathrm{GHz}$ (Giovannini et al. 2005); the non simultaneous spectral index is $\alpha_{1.6}^{5}=0.15$. The large fraction of missing flux could imply that the VLA core flux is overestimated, or that the source is variable.

$1615+35 B(N G C 6109)$ - This is a very extended Head Tail (HT) radio source with a structure more than $10^{\prime}$ in size, oriented $\mathrm{N}-\mathrm{S}$. It is associated with an elliptical galaxy of $m_{\mathrm{pg}}=14.9$ at a redshift of 0.0296 .

In our VLBA image (Fig. 19), the nuclear emission is seen with a jet on the same side as the kiloparsec-scale jet. The total flux density detected in our map is $\sim 23.6 \mathrm{mJy}$, the core flux is $\sim 17.1 \mathrm{mJy}$ and the ratio between the VLBA correlated flux and the arcsecond core flux density at $5 \mathrm{GHz}$ is $85 \%$. At 1.2 mas from the nucleus, the jet/counter-jet surface brightness ratio is $\geq 30$.

$1621+38$ - A head-tail radio galaxy (Fanti et al. 1987). In our VLBA $1.6 \mathrm{GHz}$ data it shows a core-jet structure in $\mathrm{PA} \sim 160^{\circ}$ (Fig. 20) in agreement with the $5 \mathrm{GHz}$ image of 


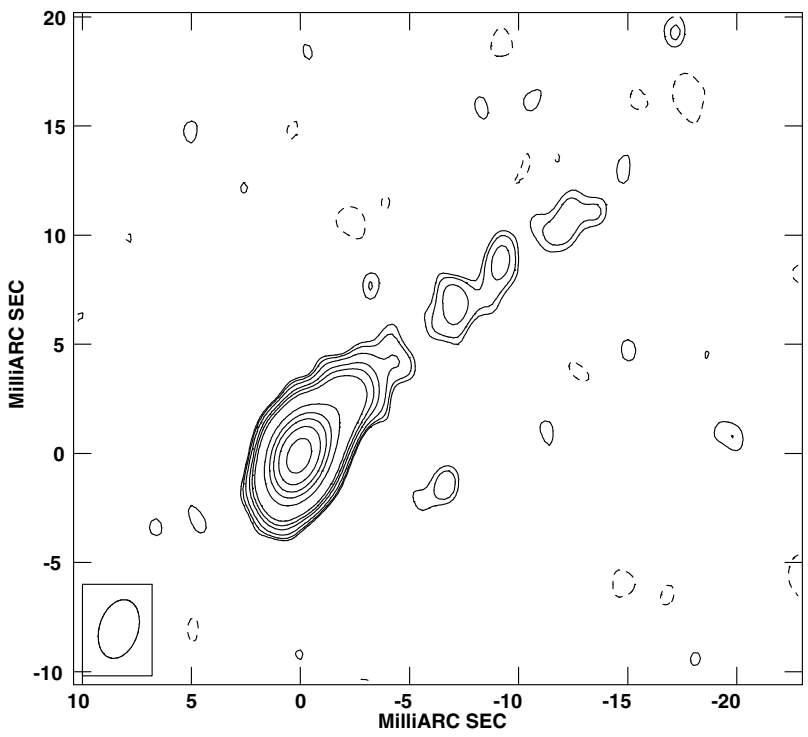

Fig. 19. VLBA image of $1615+35 \mathrm{~B}$ at $5 \mathrm{GHz}$. The HBPW is $2.8 \times$ 1.8 mas in PA $-17^{\circ}$. The noise level is $0.06 \mathrm{mJy} /$ beam and levels are $-0.150 .150 .20 .30 .50 .713571015 \mathrm{mJy} /$ beam.

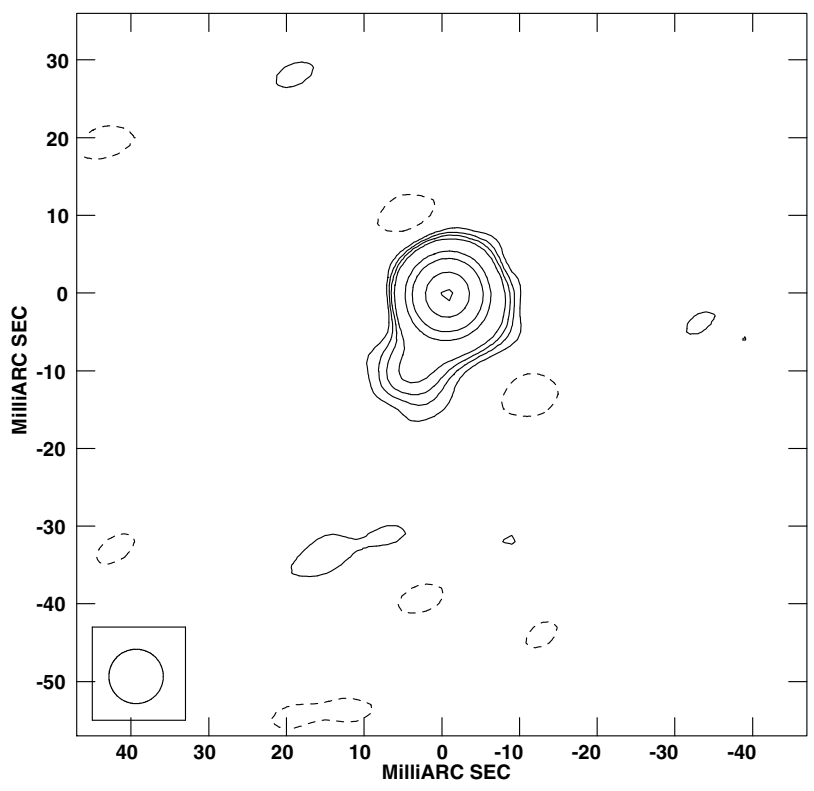

Fig. 20. VLBA image of $1621+38$ at $1.7 \mathrm{GHz}$. The HPBW is 7 mas, the noise $0.09 \mathrm{mJy} /$ beam and levels are: $-\begin{array}{llllllll}0.3 & 0.3 & 0.5 & 0.7 & 1 & 3 & 5 & 10\end{array}$ $15 \mathrm{mJy} /$ beam.

Giovannini et al. (2005). The core spectral index is $\alpha_{1.6}^{5}=0.25$ and a significant fraction of core flux density is missing.

$1637+29$ - This peculiar source displays a head-tail morphology associated with a poor group of galaxies (de Ruiter et al. 1986). The radio source consists of twin bent jets, with the main jet ending in a very bright lobe and two radio tails showing conspicuous oscillations. Our VLBA image shows an unresolved component with total flux density $S_{5} \sim 7.8 \mathrm{mJy}$. The ratio between the VLBA correlated flux and the arcsecond core flux density at $5 \mathrm{GHz}$ is $60 \%$.

$1736+32$ - The B2 source is resolved in two unrelated radio sources. The main source (the Northern one) has a FR I structure, the secondary (Southern) appears as a background FR II source projected onto the SE lobe of the FR I galaxy (Fanti et al. 1986).

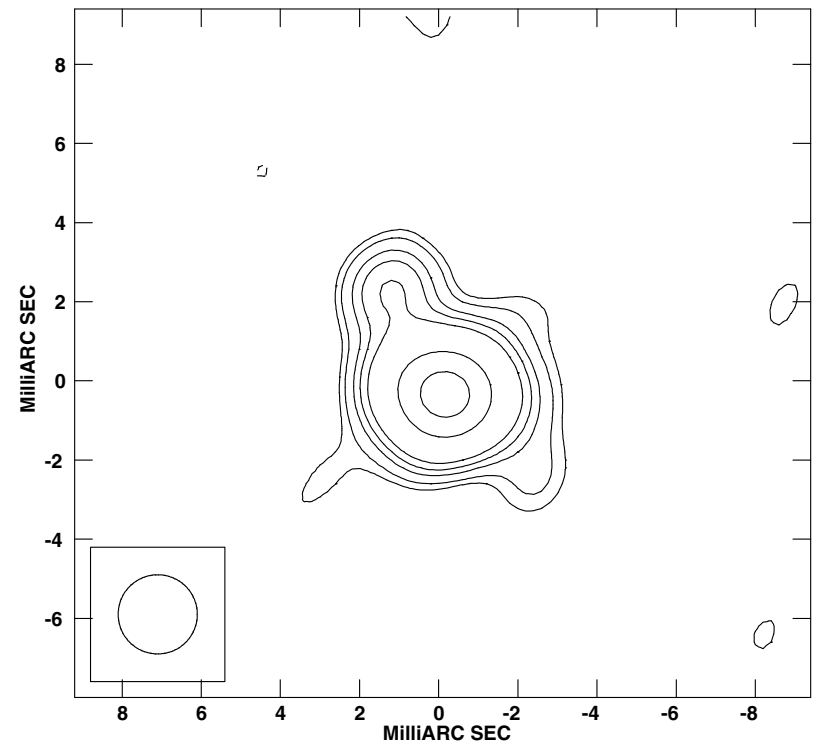

Fig. 21. VLBA image of $1752+32 \mathrm{~B}$ at $5 \mathrm{GHz}$. The HPBW is 2 mas, the noise level $0.08 \mathrm{mJy} / \mathrm{beam}$. Contours are: -0.20 .20 .30 .50 .713$ $5 \mathrm{mJy} / \mathrm{beam}$.

In our VLBA image of the main source, we found an unresolved structure with a total flux density $\sim 7.6 \mathrm{mJy}$. No arcsecond core flux is missing on the milliarcsecond scale.

$1752+32 B$ : in VLA images, this source appears as a FRI source (Capetti et al. 1993).

Our VLBA image shows nuclear emission with a two-sided emission at the same PA as the kiloparsec-scale jet (Fig. 21). The total flux density detected in our map is $\sim 9.3 \mathrm{mJy}$, the core flux is $\sim 7.4 \mathrm{mJy}$ and the ratio between the VLBA correlated flux and the arcsecond core flux density at $5 \mathrm{GHz}$ is $77 \%$.

$2236+35$ UGC 12127 - This source has a large-scale double radio jet embedded in a low-surface-brightness region with $\mathrm{S}$ symmetry (Fanti et al. 1986). In our VLBA image, the source is unresolved with total flux density $\sim 8.8 \mathrm{mJy}$ and no arcsecond core flux is missing on the milliarcsecond scale.

\section{Discussion}

\subsection{Source morphology}

Among sources with VLBI data, we have the following kiloparsec-scale structures: (a) 51 FRI radio galaxies; (b) 13 FRII radio galaxies; (c) 11 compact sources with a flat spectrum (including two BL-Lac objects), and 1 compact steep-spectrum source (CSS). The observed sample is not yet complete, but representative of a sample of sources at random angles to the line of sight. In fact, in a randomly oriented sample of radio galaxies, the probability that the source is at an angle $\theta_{1}$ and $\theta_{2}$ with respect to the line of sight is

$P\left(\theta_{1}, \theta_{2}\right)=\cos \theta_{1}-\cos \theta_{2}$.

In the observed sample, the percentage of FR I plus FR II radio sources is $84 \%$ that corresponds to sources oriented at angles greater than $33^{\circ}$, in agreement with the result obtained in Giovannini et al. (2005). We confirm with improved statistics the results discussed in Giovannini et al. (2005) and briefly review these in Sect. 1.

We note also that among sources that are compact at arcsecond resolution 3 (27\%) show a two-sided pc scale structure, 
$5(45 \%)$ are one-sided, $1(9 \%)$ is a CSO and $2(18 \%)$ have not been detected. This result confirms that low power compact sources with a flat spectrum have sub-kpc scale structure, but their small size is not always due to projection effects of beamed objects as in the case of the two BL-Lac sources and possibly the other three radio galaxies with one-sided parsec-scale structure.

The total number of sources with a two-sided jet morphology on the parsec-scale is 23 , corresponding to $30 \%$ of the sample. As discussed in Paper I (Giovannini et al. 2005), this percentage is higher than that found in previous samples in the literature: indeed, there are $11 \%(7 / 65)$ symmetric sources in the PR (Pearson-Readhead) sample (Pearson \& Readhead 1988) and 4.6\% (19/411) in the combined PR and Caltech-Jodrell (CJ) samples (Taylor et al. 1994; Polatidis et al. 2003). The main difference between the percentage of symmetric sources in the present sample and in previous samples is naturally explained in the framework of unified scheme models by the fact that the present sources show relatively faint cores and they are less affected by orientation bias. For a more detailed discussion see Giovannini et al. (2005).

We find that $27 \%$ (14) of the FR I radio galaxies and $46 \%$ (6) of the FR II radio galaxies are two-sided on the parsec scale. Assuming the same intrinsic velocity, the higher fraction of symmetric sources among FR II galaxies could suggest that the jets in FR II galaxies are intrinsically brighter than those in FR I galaxies. No correlation is found between the jet-tocounterjet ratio and the total or arcsecond core radio power.

One-sided sources are found in 31/76 sources (41\%) confirming that this structure is the most frequent pc scale structure because of Doppler boosting.

The number of unresolved sources in our VLBI images is $15(20 \%)$. Most of them (14) are FR I radio galaxies, and 1 is a FR II source $(1529+24)$. In most cases these are sources with faint relativistic jets in the plane of the sky, and therefore their jets are de-boosted.

We observed sources with a low core flux density at $5 \mathrm{GHz}$ at arcsecond resolution ( $>5 \mathrm{mJy}$ ), but only 3 sources ( $4 \%$ ) have not been detected in our VLBI observations. Two of them are C-shaped sources as discussed before, and the other one is a FR I radio galaxy. This result confirms that also in low power sources and in radio sources with a low radio nuclear activity a compact nuclear radio source is present.

Finally we would like to discuss the two radio sources where a Z-shaped structure is present on the parsec-scale. At present among BCS sources these are 1502+26 (3C310), and 1346+26 (4C26.42). A few other are present in literature as e.g. the CSO 1946+708 (Taylor et al. 2009). The presence of this symmetric and large change in the jet direction so near the core cannot be due to projection or relativistic effects. The large symmetry present in these sources imply that are oriented close to the plane of the sky, moreover a change in the jet velocity and/or orientation would produce a change in the Doppler factor that should be revealed by the observations of the jet properties. As discussed in more detail in Liuzzo et al. (2009) for $1346+26$ the source morphology strongly suggests that in these sources the jets are not highly relativistic even at a few pc distance from the core. A slow and possibly high density jet could more easily interact with a turbulent surrounding medium in rotation near the central Black Hole to show this peculiar symmetric structure.

\subsection{Missing flux in parsec scale structures}

We compared the total flux at VLBA scale with the core arcsecond flux density (Table 4). Over all data, among 76 sources, we find that $47(62 \%)$ have a correlated flux density larger than $70 \%$ of the arcsecond core flux density. This means that in these sources we mapped most of the mas scale structure and so we can properly connect the parsec to the kiloparsec structures. Instead, for 19 (38\%) sources, a significant fraction of the arcsecond core flux density is missing in the VLBA images. This suggests variability or the presence of significant structures between $\sim 30$ mas and 1 arcsec that the VLBA can miss due to a lack of short baselines. In particular among the 6 sources where the VLBA flux density is less than $10 \%$ of the arcsecond core flux density measured by the VLA, we have evidence of a complex structure in agreement with an intermediate-scale structure lost in our images. Variability should be also considered. To properly study these structures, future observations with the EVLA at high frequency or with the e-MERLIN array will be necessary.

\subsection{Jet velocity and orientation}

For 51 sources we can estimate the ratio between the jet and counter-jet brightness or provide a lower limit to the ratio (Table 4). Among the 25 sources with a ratio $\gtrsim 10$ we have one-sided sources with the exception of $0055+30$ and $0220+43$ where deep observations allowed us to detect a counterjet structure despite a large brightness ratio. The presence of large ratios confirms the presence of relativistic jets.

We also estimated the core dominance of observed sources (Table 4). According to Giovannini et al. (1994) and references therein, a correlation is present between the core and total radio power and we can use the core dominance to estimate the jet velocity and orientation. In Table 4 we present the core dominance defined as the ratio between the observed and the estimated core radio power according to the relation given in Giovannini et al. (1994).

A ratio larger than 1 implies a source with a boosted core, therefore oriented at an angle smaller than $60^{\circ}$. For a value of the core dominance in the range $0.25-1$, the core radio power is lower than the value expected from the correlation therefore the nuclear observed power is de-boosted and the source is oriented at an angle larger than $60^{\circ}$. These values are in general in agreement with the ratio between the jet and counterjet brightness and can give useful constraints on the jet velocity and orientation. Three sources show a core dominance larger than 10: two BL Lacs and 0222+36, a symmetric source in VLBI images but with evidence of restarted activity (Giroletti et al. 2005a,b).

If the core dominance is smaller than 0.25 no solution can be found from the correlation. Such a strong deboosting cannot be obtained even if the source is in the plane of the sky and the jets are strongly relativistic. We interpret such a result as a possible nuclear variability with the core in a low activity regime and/or the evidence that the nuclear activity is now stopped and the total radio power is related to a previous episode of greater nuclear activity, i.e. the AGN is dying. We have 8 sources with a such low core dominance. Four of them are FR I radio galaxies $(0300+16,1122+39,1257+28,1357+28)$ characterized by a faint nuclear emission in VLA observations at $5 \mathrm{GHz}$ not only with respect to the total low frequency flux density, but also with respect to the source total flux density at the same frequency. The remaining four sources are narrow line extended and powerful FR II radio galaxies $(0106+13,0356+10,0802+24,1319+42)$, lobe dominated and with evidence of spectral steepening at high frequency. Also in these sources the extended radio lobes suggest a previous episode of greater nuclear activity. 


\section{Conclusion}

In this paper, we continue the study of the BCS sample. We present here new VLBA data for 33 sources, we briefly discuss the parsec and kiloparsec-scale structures, and we summarise the results obtained to date.

- The detection rate is high: only 3 sources out of 76 (4\%) have not been detected, even though we observed sources with an arcsecond core flux density as low as $5 \mathrm{mJy}$ at $5 \mathrm{GHz}$. This result confirms the presence of compact radio nuclei at the center of radio galaxies.

- As expected in sources with relativistic parsec-scale jets, the one-sided jet morphology is the predominant structure present in our VLBI images, however $22 \%$ of the observed sources show evidence of a two-sided strucure. This result is in agreement with a random orientation and a high jet velocity $(\beta \sim 0.9)$.

- We find two sources with a Z-shaped structure on the parsecscale suggesting the presence of low velocity jets, in these peculiar radio sources.

- In 8 sources the low core dominance suggests that the nuclear activity is now in a low activity state. The dominance of the extended emission implies a greater activity of the core in the past. However in these sources a parsec-scale core and even jets are present. In this scenario the nuclear activity may be in a low or high state but is not completely quiescent. This result is in agreement with the evidence that a few sources show evidence of a recurring or re-starting activity. This point can be better addressed when observations are available for the full sample so that we can discuss the time-scale of the recurring activity.

- In most cases, the parsec and the kiloparsec scale jet structures are aligned and the main jet is always on the same side with respect to the nuclear emission. This confirms the idea that the large bends present in some BL Lacs sources are amplified by the small jet orientation angle with respect to the line-of-sight.

- In $62 \%$ of the sources, there is good agreement between the arcsecond-scale and the VLBI correlated flux density. For the other $38 \%$ of the sources, at the milliarcsecond scale more than $30 \%$ of the arcsecond core flux density is missing. This suggests the presence of variability, or of a significant subkiloparsec-scale structure, which will be better investigated with the EVLA at high frequency or with the e-MERLIN array.

- To complete VLBI observations of the BCS sample, 18 sources of the sample will be observed with VLBI in the future. Since these sources have a very faint nuclear emission in the radio band we will require very sensitive VLBI observations (employing large bandwidths, long integration times, and phase referencing).

Acknowledgements. This research has made use of the NASA-IPAC Extragalactic Data Base (NED) which is operated by the JPL, California Institute of Technology, under contract with the National Aeronautics and Space Administration.

\section{References}

Baum, S. A., Heckman, T. M., Bridle, A., van Breugel, W. J. M., \& Miley, G. K. 1988, ApJS, 68, 643

Baum, S. A., Heckman, T., \& van Breugel, W. 1990, ApJS, 74, 389

Beswick, R. J., Peck, A. B., Taylor, G. B., \& Giovannini, G. 2004, MNRAS, 352, 49

Blandford, R. D., \& Icke, V. 1978, MNRAS, 185527

Blanton, E. L., Sarazin, C. L., McNamara, B. R., \& Clarke, T. E. 2004, ApJ, 612, 817

Burns, J. O., Gregory, S. A., \& Holman, G. D. 1981, ApJ, 250, 450 Capetti, A., Morganti, R., Parma, P., \& Fanti, R. 1993, A\&AS, 99, 407

Capetti, A., de Ruiter, H. R., Fanti, R., et al. 2000, A\&A, 362, 871 Cotton, W. D. 1993, AJ, 106, 1241

de Ruiter, H. R., Parma, P., Fanti, C., \& Fanti, R. 1986, A\&AS, 65, 111

Fanti, C., Fanti, R., de Ruiter, H. R., \& Parma, P. 1986, A\&AS, 65, 145 Fanti, C., Fanti, R., de Ruiter, H. R., \& Parma, P. 1987, A\&AS, 69, 57

Feretti, L., Giovannini, G., Gregorini, L., Parma, P., \& Zamorani, G. 1984, A\&A, 139,55

Floyd, D. J. E., Perlman, E., Leahy, J. P., et al. 2006, ApJ, 639, 23 Gentile, G., Rodríguez, C., Taylor, G. B., et al. 2007, ApJ, 659, 225

Giovannini, G., Feretti, L., Gregorini, L., \& Parma, P. 1988, A\&A, 199, 73

Giovannini, G., Feretti, L., \& Comoretto, G. 1990, ApJ, 358, 159

Giovannini, G., Feretti, L., Venturi, T., et al. 1994, ApJ, 435, 116

Giovannini, G., Cotton, W. D., Feretti, L., Lara, L., \& Venturi, T. 2001, ApJ, 552, 508

Giovannini, G., Taylor, G. B., Feretti, L., et al. 2005, ApJ, 618, 635

Giovannini, G., Giroletti, M., \& Taylor, G. B. 2007, A\&A, 474, 409

Giroletti, M., Giovannini, G., Taylor, G. B., et al. 2003a, A\&A 399, 889

Giroletti, M., Giovannini, G., Taylor, G. B., \& Conway, J. E. 2003b, New Astron. Rev., 47, 613

Giroletti, M., Giovannini, G., Feretti, L., et al. 2004, ApJ, 600, 127

Giroletti, M., Taylor, G. B., \& Giovannini, G. 2005a, ApJ, 622, 178

Giroletti, M., Giovannini, G., \& Taylor, G. B. 2005b, A\&A, 441, 89

Giroletti, M., Giovannini, G., Cotton, W. D., et al. 2008, A\&A, 488, 905

Giroletti, M., et al. in preparation.

Gizani, N. A. B., Garrett, M. A., Morganti, R., et al. 2002, evn..conf, 163

Gonzalez-Serrano, J. I., Carballo, R., \& Perez-Fournon, I. 1993, AJ, 105, 1710

Hardcastle, M. J., Alexander, P., Pooley, G. G., \& Riley, J. M. 1998, MNRAS, 296,445

Jamrozy, M., Konar, C., Saikia, D. J., et al. 2007, MNRAS, 378, 581

Jetha, N. N., Hardcastle, M. J., Ponman, T. J., \& Sakelliou, I. 2008, MNRAS, in press [arXiv:0809.2534]

Lara, L., Giovannini, G., Cotton, W. D., Feretti, L., \& Venturi, T. 2004, A\&A, 415,905

Leahy, J. P., \& Williams, A. G. 1984, MNRAS, 210, 929

Leahy, J. P., \& Perley, R. A. 1991, AJ, 102, 537

Ledlow, M. J., Voges, W., Owen, F. N., \& Burns, J. O. 2003, AJ, 126, 2740

Liuzzo, E., Taylor, G. B., Giovannini, G., \& Giroletti, M. 2009, A\&A, 501, 933 Machalski, J. 1998, A\&AS, 128, 153

Massaro, F., Chiaberge, M., Grandi, P., et al. 2009, ApJ, 692, L123

Murgia, M., Parma, P., de Ruiter, H. R., et al. 2001, A\&A, 380, 102

Parma, P., Ekers, R. D., \& Fanti, R. 1985, A\&AS, 59, 511

Parma, P., de Ruiter, H. R., Fanti, C., \& Fanti, R. 1986, A\&AS, 64, 135

Pearson T. J., \& Readhead, A. C. S. 1988, ApJ, 328, 114

Pearson, T. J., Shepherd, M. C., Taylor, G. B., \& Myers, S. T. 1994, BAAS, 26, 1318

Polatidis, A. G., Wilkinson, P. N., Xu, W., et al. 1995, ApJS, 98, 1

Roche, N., \& Eales, S. A. 2000, MNRAS, 317, 120

Sandage, A. 1972, ApJ, 178, 25

Taylor, G. B., \& Vermeulen, R. C. 1997, ApJ, 485, L9

Taylor, G. B., Vermeulen, R. C., Pearson, T. J., et al. 1994, ApJS, 95, 345

Taylor, G. B., Charlot, P., Vermeulen, R. C., \& Pradel, N. 2009 ApJ, in press [arXiv:0904.1879]

Trussoni, E., Massaglia, S., Ferrari, R., et al. 1997, A\&A, 327, 27

Valentijn, E. A. 1979, A\&A, 78, 367

Vallèe, J. P. 1982, AJ, 87, 486

van Breugel, W., \& Fomalont, E. B. 1984, ApJ, 282, L55

van Breugel, W., Miley, G., Heckman, T., Butcher, H., \& Bridle, A. 1985, ApJ, 290, 496

van Breugel, W. J. M., Heckman, T. M., Miley, G. K., \& Filippenko, A. V. 1986, ApJ, 311, 58

Venturi, T., Castaldini, C., Cotton, W. D., et al. 1995, ApJ, 454, 735 
E. Liuzzo et al.: The Bologna complete sample of nearby radio sources. II., Online Material $p 1$

Table 1. The complete bologna sample.

\begin{tabular}{|c|c|c|c|c|c|c|c|}
\hline $\begin{array}{l}\text { Name } \\
\text { IAU }\end{array}$ & $\begin{array}{l}\text { Name } \\
\text { other }\end{array}$ & 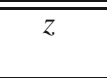 & $\begin{array}{c}\text { Morphology } \\
\text { kpc }\end{array}$ & $\begin{array}{l}S_{\mathrm{c}}(5.0) \\
\mathrm{mJy}\end{array}$ & $\begin{array}{r}\bar{L} \log P_{\mathrm{c}} \\
\mathrm{W} / \mathrm{Hz}\end{array}$ & $\begin{array}{l}\log P_{\mathrm{t}} \\
\mathrm{W} / \mathrm{Hz}\end{array}$ & Notes \\
\hline $0034+25$ & UGC 367 & 0.0321 & FR I & 10 & 22.40 & 23.80 & $\mathrm{~N}$ \\
\hline $0055+26$ & N326 & 0.0472 & FR I & 11 & 22.75 & 25.40 & $\mathrm{~N}$ \\
\hline $0055+30$ & N315 & 0.0167 & FR I & 588 & 23.57 & 24.24 & G \\
\hline $0104+32$ & $3 C 31$ & 0.0169 & FR I & 92 & 22.76 & 24.80 & G \\
\hline $0106+13$ & $3 \mathrm{C} 33$ & 0.0595 & FR II & 24 & 23.30 & 26.42 & I \\
\hline $0116+31$ & 4C 31.04 & 0.0592 & $\mathrm{C}$ & 1250 & 23.42 & 25.46 & I, 1 \\
\hline $0120+33$ & N507 & 0.0164 & FR I & 1.4 & 20.93 & 23.62 & no VLBI \\
\hline $0149+35$ & N708 & 0.0160 & FR I & 5 & 21.46 & 23.32 & $\mathrm{~N}$ \\
\hline $0206+35$ & 4C 35.03 & 0.0375 & FR I & 106 & 23.54 & 25.17 & G \\
\hline $0220+43$ & $3 \mathrm{C} 66 \mathrm{~B}$ & 0.0215 & FR I & 182 & 23.28 & 25.30 & G \\
\hline $0222+36$ & & 0.0327 & $\mathrm{C}$ & 140 & 23.52 & 23.93 & $\mathrm{G}, 5 \mathrm{~b}$ \\
\hline $0258+35$ & N1167 & 0.0160 & CSS & $<243$ & $<23.14$ & 24.35 & $\mathrm{G}, 5 \mathrm{~b}$ \\
\hline $0300+16$ & 3С 76.1 & 0.0328 & FR I & 10 & 21.38 & 24.09 & $\mathrm{~N}$ \\
\hline $0326+39$ & & 0.0243 & FR I & 78 & 23.01 & 24.40 & I \\
\hline $0331+39$ & 4C 39.12 & 0.0202 & $\mathrm{C}$ & 149 & 23.11 & 24.20 & G \\
\hline $0356+10$ & $3 \mathrm{C} 98$ & 0.0306 & FR II & 9 & 22.28 & 25.73 & $\mathrm{~N}$ \\
\hline $0648+27$ & & 0.0409 & $\mathrm{C}$ & 58 & 23.36 & 24.02 & $\mathrm{G}, 5 \mathrm{~b}$ \\
\hline $0708+32$ & & 0.0672 & FR I & 15 & 23.20 & 24.49 & $\mathrm{~N}$ \\
\hline $0722+30$ & & 0.0191 & Spiral? & 51 & 22.62 & 23.49 & $\mathrm{~N}$ \\
\hline $0755+37$ & N2484 & 0.0413 & FR I & 195 & 23.82 & 25.36 & G \\
\hline $0800+24$ & & 0.0433 & FR I & 3 & 22.11 & 24.14 & no VLBI \\
\hline $0802+24$ & 3C 192 & 0.0597 & FR II & 8 & 22.83 & 26.01 & $\mathrm{~N}$ \\
\hline $0828+32 \mathrm{AB}$ & 4C 32.15 & 0.0507 & FR II & 3.3 & 22.29 & 25.41 & no VLBI \\
\hline 0836+29-I & 4C 29.30 & 0.0650 & FR I & 63.0 & 23.79 & 25.19 & $\mathrm{~N}$ \\
\hline 0836+29-II & 4C 29.30 & 0.0790 & FR I & 131 & 24.28 & 25.42 & G \\
\hline $0838+32$ & 4C 32.26 & 0.0680 & FR I & 7.5 & 22.91 & 25.32 & $\mathrm{~N}$ \\
\hline $0844+31$ & IC 2402 & 0.0675 & FR II & 40 & 23.56 & 25.53 & I \\
\hline $0913+38$ & & 0.0711 & FR I & $<1.0$ & $<22.08$ & 24.99 & no VLBI \\
\hline $0915+32$ & & 0.0620 & FR I & 8.0 & 22.86 & 24.63 & $\mathrm{~N}$ \\
\hline $0924+30$ & & 0.0266 & FR I & $<0.4$ & $<20.80$ & 24.44 & no VLBI \\
\hline $1003+35$ & $3 C 236$ & 0.0989 & FR II & 400 & 24.97 & 26.16 & I \\
\hline $1037+30$ & 4C 30.19 & 0.0909 & $\mathrm{C}$ & $<84$ & $<24.22$ & 25.36 & I \\
\hline $1040+31$ & & 0.0360 & $\mathrm{C}$ & 55 & 23.21 & 24.70 & I \\
\hline $1101+38$ & Mkn 421 & 0.0300 & BL Lac & 640 & 24.11 & 24.39 & G \\
\hline $1102+30$ & & 0.0720 & FR I & 26 & 23.50 & 25.07 & I \\
\hline $1113+29$ & 4C 29.41 & 0.0489 & FR I & 41 & 23.27 & 25.35 & $\mathrm{~N}$ \\
\hline $1116+28$ & & 0.0667 & FR I & 30 & 23.50 & 25.05 & $\mathrm{I}, \mathrm{N}^{*}$ \\
\hline $1122+39$ & N3665 & 0.0067 & FR I & 6 & 20.77 & 22.45 & $\mathrm{~N}$ \\
\hline $1142+20$ & $3 C 264$ & 0.0206 & FR I & 200 & 23.28 & 25.17 & $\mathrm{G}, 3$ \\
\hline $1144+35$ & & 0.0630 & FR I & 250 & 24.37 & 24.49 & $\mathrm{G}, 7$ \\
\hline $1204+24$ & & 0.0769 & FR I & 8 & 23.05 & 24.58 & $\mathrm{~N}$ \\
\hline $1204+34$ & & 0.0788 & FR II? & 23 & 23.53 & 25.17 & I \\
\hline $1217+29$ & N4278 & 0.0021 & $\mathrm{C}$ & 162 & 21.20 & 21.75 & G, 5 \\
\hline $1222+13$ & $3 C 272.1$ & 0.0037 & FR I & 180 & 21.74 & 23.59 & G \\
\hline $1228+12$ & $3 \mathrm{C} 274$ & 0.0037 & FR I & 4000 & 23.30 & 25.38 & G \\
\hline $1243+26 B$ & & 0.0891 & FR I & $<1.8$ & $<22.54$ & 25.23 & no VLBI \\
\hline $1251+27$ & $3 C 277.3$ & 0.0857 & FR II & 12 & 23.33 & 26.05 & $\mathrm{~N}$ \\
\hline $1254+27$ & N4839 & 0.0246 & FR I & 1.5 & 21.31 & 23.45 & 9 \\
\hline $1256+28$ & N4869 & 0.0224 & FR I & 2.0 & 21.31 & 24.22 & no VLBI \\
\hline $1257+28$ & N4874 & 0.0239 & FR I & 1.1 & 21.15 & 23.81 & 9 \\
\hline $1316+29$ & 4C 29.47 & 0.0728 & FR I & 31 & 23.58 & 25.59 & I \\
\hline $1319+42$ & $3 C 285$ & 0.0797 & FR I/II & 6 & 22.95 & 25.92 & $\mathrm{~N}$ \\
\hline $1321+31$ & N5127 & 0.0161 & FR I & 21 & 22.09 & 24.32 & I \\
\hline $1322+36$ & N5141 & 0.0175 & FR I & 150 & 23.01 & 24.07 & G \\
\hline $1339+26$ & UGC 8669 & 0.0722 & FR I & $<55$ & $<23.83$ & 25.19 & $\mathrm{~N}$ \\
\hline $1346+26$ & 4C 26.42 & 0.0633 & FR I & 53 & 23.70 & 25.47 & $\mathrm{I}, \mathrm{N}^{*}, 8$ \\
\hline $1347+28$ & & 0.0724 & FR I & 4.8 & 22.78 & 24.81 & no VLBI \\
\hline $1350+31$ & $3 C 293$ & 0.0452 & FR I & $<100$ & $<23.67$ & 25.68 & $\mathrm{I}, \mathrm{N}^{*}, 4$ \\
\hline $1357+28$ & & 0.0629 & FR I & 6.2 & 21.97 & 24.81 & $\mathrm{~N}$ \\
\hline $1414+11$ & 3С 296 & 0.0237 & FR I & 77 & 22.98 & 24.93 & I \\
\hline $1422+26$ & & 0.0370 & FR I & 25.0 & 22.89 & 24.80 & I \\
\hline $1430+25$ & & 0.0813 & FR I & $<1.0$ & $<22.20$ & 25.34 & no VLBI \\
\hline $1441+26$ & & 0.0621 & FR I & $<0.7$ & $<21.81$ & 24.78 & no VLBI \\
\hline
\end{tabular}


E. Liuzzo et al.: The Bologna complete sample of nearby radio sources. II., Online Material p 2

Table 1. continued.

\begin{tabular}{|c|c|c|c|c|c|c|c|}
\hline $\begin{array}{l}\text { Name } \\
\text { IAU }\end{array}$ & $\begin{array}{l}\text { Name } \\
\text { other }\end{array}$ & $\bar{z}$ & $\begin{array}{c}\text { Morphology } \\
\text { kpc }\end{array}$ & $\begin{array}{l}S_{\mathrm{c}}(5.0) \\
\mathrm{mJy}\end{array}$ & $\begin{array}{r}\log P_{\mathrm{c}} \\
\mathrm{W} / \mathrm{Hz}\end{array}$ & $\begin{array}{l}\overline{\log P_{\mathrm{t}}} \\
\mathrm{W} / \mathrm{Hz}\end{array}$ & Notes \\
\hline $1448+63$ & $3 \mathrm{C} 305$ & 0.0410 & FR I & 29 & 23.05 & 25.44 & $\bar{I}$ \\
\hline $1502+26$ & $3 C 310$ & 0.0540 & FR I & 80 & 23.73 & 26.20 & $\mathrm{I}, \mathrm{N}^{*}$ \\
\hline $1512+30$ & & 0.0931 & $\mathrm{C}$ & $<0.4$ & $<21.92$ & 24.75 & no VLBI \\
\hline $1521+28$ & & 0.0825 & FR I & 40 & 23.81 & 25.40 & I \\
\hline $1525+29$ & & 0.0653 & FR I & 2.5 & 22.40 & 24.64 & no VLBI \\
\hline $1528+29$ & & 0.0843 & FR I & 4.5 & 22.89 & 25.10 & no VLBI \\
\hline $1529+24$ & 3C 321 & 0.0960 & FR II & 30 & 23.83 & 26.20 & I \\
\hline $1549+20$ & $3 \mathrm{C} 326$ & 0.0895 & FR II & 3.5 & 22.83 & 26.27 & no VLBI \\
\hline $1553+24$ & & 0.0426 & FR I & 40 & 23.22 & 23.92 & I \\
\hline $1557+26$ & IC 4587 & 0.0442 & $\mathrm{C}$ & 31 & 23.42 & 24.05 & I \\
\hline $1610+29$ & N6086 & 0.0313 & FR I & $<6.0$ & $<22.12$ & 23.85 & no VLBI \\
\hline $1613+27$ & & 0.0647 & FR I & 25 & 23.40 & 24.65 & I \\
\hline $1615+35 B$ & N6109 & 0.0296 & FR I & 28 & 22.75 & 25.13 & $\mathrm{~N}$ \\
\hline $1621+38$ & N6137 & 0.0310 & FR I & 50 & 23.03 & 24.30 & I \\
\hline $1626+39$ & 3C 338 & 0.0303 & FR I & 105 & 23.33 & 25.57 & $\mathrm{G}, 6$ \\
\hline $1637+29$ & & 0.0875 & FR I & 13 & 23.38 & 25.17 & $\mathrm{~N}$ \\
\hline $1652+39$ & Mkn 501 & 0.0337 & BL-Lac & 1250 & 24.51 & 24.36 & G, 2 \\
\hline $1657+32 \mathrm{~A}$ & & 0.0631 & FR I & 2.5 & 22.37 & 25.07 & no VLBI \\
\hline $1658+30$ & 4C 30.31 & 0.0351 & FR I & 84 & 23.36 & 24.63 & I \\
\hline $1736+32$ & & 0.0741 & FR I & 8 & 23.01 & 24.89 & $\mathrm{~N}$ \\
\hline $1752+32 B$ & & 0.0449 & FR I & 12 & 22.74 & 24.17 & $\mathrm{~N}$ \\
\hline $1827+32 \mathrm{~A}$ & & 0.0659 & FR I & 26 & 23.43 & 24.88 & I \\
\hline $1833+32$ & 3C 382 & 0.0586 & FR II & 188 & 24.18 & 26.04 & G \\
\hline $1842+45$ & 3C 388 & 0.0917 & FR II & 62 & 24.10 & 26.47 & I \\
\hline $1845+79$ & $3 C 390.3$ & 0.0569 & FR II & 330 & 24.39 & 26.30 & G \\
\hline $1855+37$ & & 0.0552 & $\mathrm{C}$ & $<100$ & $<23.85$ & 24.75 & I \\
\hline $2116+26$ & N7052 & 0.0164 & FR I & 47 & 22.46 & 23.29 & I \\
\hline $2212+13$ & $3 \mathrm{C} 442$ & 0.0262 & FR I? & 2.0 & 21.49 & 25.21 & no VLBI \\
\hline $2229+39$ & 3C 449 & 0.0181 & FR I & 37 & 22.44 & 24.68 & I \\
\hline $2236+35$ & UGC 12127 & 0.0277 & FR I & 8.0 & 22.14 & 24.12 & $\mathrm{~N}$ \\
\hline $2243+39$ & $3 \mathrm{C} 452$ & 0.0811 & FR II & 130 & 24.30 & 26.64 & G \\
\hline $2335+26$ & $3 \mathrm{C} 465$ & 0.0301 & FR I & 270 & 23.74 & 25.61 & G \\
\hline
\end{tabular}

$S_{\mathrm{c}}(5.0)$ is the arcsecond core flux density at $5.0 \mathrm{GHz}$;

$\log P_{\mathrm{c}}$ is the corresponding logarithm of the radio power;

$\log P_{\mathrm{t}}$ is the $\log$ arithm of the total radio power at $408 \mathrm{MHz}$;

Morphology: $\mathrm{C}=$ flat spectrum compact core, $\mathrm{CSO}=$ Compact Symmetric Object, $\mathrm{CSS}=$ Compact Steep Spectrum source,

FRI or II = Fanaroff types I or II;

Notes refer to the status of VLBI observations:

G: Giovannini et al. (2001); I: Giovannini et al. (2005); N, N* new data are given in this paper;

1: Giroletti et al. (2003a,b); 2: Giroletti et al. (2004, 2008) 3: Lara et al. (2004) 4: Beswick et al. (2004);

5: Giroletti et al. (2005) 5b: Giroletti et al. (2005b) 6: Gentile et al. 2007);

7: Giovannini et al. (2007); 8: Liuzzo et al. (2009), submitted; 9: Liuzzo et al. (2009), in preparation;

For a reference to the extended structure see Fanti et al. (1987) for B2 sources and Leahy et al. at the URL: http://www.jb.man.ac.uk/ atlas). 
E. Liuzzo et al.: The Bologna complete sample of nearby radio sources. II., Online Material $p 3$

Table 2. Sources and calibrators list.

\begin{tabular}{|c|c|c|c|c|}
\hline \multirow{2}{*}{$\begin{array}{l}\text { Name } \\
\text { IAU }\end{array}$} & \multicolumn{2}{|c|}{ Core absolute position } & \multirow{2}{*}{$\begin{array}{l}\text { Phase } \\
\text { calibrator }\end{array}$} & \multirow{2}{*}{$\begin{array}{l}\text { ATMCA } \\
\text { calibrators }\end{array}$} \\
\hline & $\begin{array}{c}\mathrm{RA}_{(J 2000)} \\
\left({ }^{\mathrm{hms}}\right)\end{array}$ & $\begin{array}{c}\operatorname{Dec}_{J 2000} \\
\left({ }^{\prime \prime}, \prime\right)\end{array}$ & & \\
\hline $0034+25$ & 003705.488 & 254156.331 & $\mathrm{~J} 0046+2456$ & $\mathrm{~J} 0106+2539, \mathrm{~J} 0057+3021, \mathrm{~J} 0019+2602$ \\
\hline $0055+26$ & 005822.630 & 265158.689 & J0046+2456 & $\mathrm{J} 0106+2539, \mathrm{~J} 0057+3021, \mathrm{~J} 0019+2602$ \\
\hline $0149+35$ & 015246.458 & 360906.494 & J0152+3716 & $\mathrm{J} 0137+3309, \mathrm{~J} 0205+3212$ \\
\hline $0300+16$ & 030315.014 & 162618.978 & J0305+1734 & $\mathrm{J} 0256+1334, \mathrm{~J} 0321+1221$ \\
\hline $0356+10$ & 035854.437 & 102602.778 & J0409+1217 & $\mathrm{J} 0407+0742, \mathrm{~J} 0345+1453$ \\
\hline $0708+32 B$ & 071147.669 & 321835.946 & J0714+3534 & J0646+3041 \\
\hline $0722+30$ & ND & ND & J0736+2954 & J0646+3041 \\
\hline $0802+24$ & 080535.005 & 240950.329 & $\mathrm{~J} 0802+2509$ & $\mathrm{~J} 0813+2542, \mathrm{~J} 0805+2106$ \\
\hline $0836+29-\mathrm{I}$ & 084002.352 & 294902.529 & J0839+2850 & $\mathrm{J} 0827+3525, \mathrm{~J} 0852+2833, \mathrm{~J} 0823+2928$ \\
\hline $0838+32$ & 084113.097 & 322459.712 & J0839+2850 & $\mathrm{J} 0827+3525, \mathrm{~J} 0852+2833, \mathrm{~J} 0823+2928$ \\
\hline $0915+32 B$ & 091859.406 & 315140.636 & J0919+3324 & J0915+2933 \\
\hline $1113+29$ & 111634.619 & 291517.121 & $\mathrm{~J} 1125+2610$ & $\mathrm{~J} 1130+3031, \mathrm{~J} 1103+3014, \mathrm{~J} 1102+2757$ \\
\hline \multirow[t]{2}{*}{$1116+28$} & 111859.366 & 275407.003 & $\mathrm{~J} 1125+2610$ & $\mathrm{~J} 1130+3031, \mathrm{~J} 1103+3014, \mathrm{~J} 1102+2757$ \\
\hline & 111859.366 & 275407.002 & $\mathrm{~J} 1125+2610$ & $1.6 \mathrm{GHz}$ data \\
\hline $1122+39$ & 112443.624 & 384546.278 & $\mathrm{~J} 1130+3815$ & $\mathrm{~J} 1104+3812, \mathrm{~J} 1108+4330$ \\
\hline $1204+24$ & 120707.365 & 235424.886 & J1209+2547 & $\mathrm{J} 1150+2417, \mathrm{~J} 1212+1925$ \\
\hline $1251+27 B$ & 125412.007 & 273733.961 & $\mathrm{~J} 1300+2830$ & $\mathrm{~J} 1230+2518$ \\
\hline $1319+42$ & 132117.867 & 423514.989 & $\mathrm{~J} 1327+4326$ & $\mathrm{~J} 1324+4048, \mathrm{~J} 1254+4536$ \\
\hline $1339+26 B$ & ND & ND & $\mathrm{J} 1342+2709$ & $\mathrm{~J} 1327+2210 \mathrm{~J} 1407+2827, \mathrm{~J} 1329+3154$ \\
\hline \multirow{2}{*}{$1346+26$} & 134852.489 & 263534.341 & $\mathrm{~J} 1342+2709$ & $\mathrm{~J} 1327+2210, \mathrm{~J} 1407+2827, \mathrm{~J} 1329+3154$ \\
\hline & 134852.489 & 263534.337 & $\mathrm{~J} 1350+3034$ & $1.6 \mathrm{GHz}$ data \\
\hline $1350+31$ & 135217.801 & 312646.463 & $\mathrm{~J} 1350+3034$ & $1.6 \mathrm{GHz}$ data \\
\hline $1357+28$ & 140000.854 & 282959.801 & $\mathrm{~J} 1342+2709$ & $\mathrm{~J} 1327+2210, \mathrm{~J} 1407+2827, \mathrm{~J} 1329+3154$ \\
\hline $1422+26$ & 142440.529 & 263730.476 & $\mathrm{~J} 1436+2321$ & $1.6 \mathrm{GHz}$ data \\
\hline $1448+63$ & 144921.61 & 631614.3 & $\mathrm{~J} 1441+6318$ & $1.6 \mathrm{GHz}$ data \\
\hline \multirow[t]{2}{*}{$1502+26$} & 150457.120 & 260058.455 & $\mathrm{~J} 1453+2648$ & $\mathrm{~J} 1522+3144, \mathrm{~J} 1516+1932$ \\
\hline & 150457.109 & 260058.473 & $\mathrm{~J} 1453+2648$ & $1.6 \mathrm{GHz}$ data \\
\hline $1529+24$ & 153143.47 & 240419.1 & J1539+2744 & $1.6 \mathrm{GHz}$ data \\
\hline $1557+26$ & 155951.614 & 255626.319 & $\mathrm{~J} 1610+2414$ & $1.6 \mathrm{GHz}$ data \\
\hline $1613+27$ & 161531.360 & 272657.335 & $\mathrm{~J} 1610+2414$ & $1.6 \mathrm{GHz}$ data \\
\hline $1615+35 B$ & 161740.537 & 350015.201 & $\mathrm{~J} 1613+3412$ & $\mathrm{~J} 1635+3808, \mathrm{~J} 1642+2523$ \\
\hline $1621+38$ & 162303.119 & 375520.552 & $\mathrm{~J} 1640+3946$ & 1.6 GHz data \\
\hline $1637+29$ & 163920.117 & 295055.855 & $\mathrm{~J} 1653+3107$ & $\mathrm{~J} 1635+3808, \mathrm{~J} 1642+2523$ \\
\hline $1736+32$ & 173835.770 & 325601.524 & $\mathrm{~J} 1738+3224$ & $\mathrm{~J} 1735+3616, \mathrm{~J} 1753+2848$ \\
\hline $1752+32 B$ & 175435.503 & 323417.192 & $\mathrm{~J} 1748+3404$ & $\mathrm{~J} 1735+3616, \mathrm{~J} 1753+2848$ \\
\hline $2236+35$ & 223829.421 & 351946.872 & $\mathrm{~J} 2248+3718$ & $\mathrm{~J} 2216+3518, \mathrm{~J} 2236+2828$ \\
\hline
\end{tabular}


E. Liuzzo et al.: The Bologna complete sample of nearby radio sources. II., Online Material p 4

Table 3. VLBA observations.

\begin{tabular}{|c|c|c|c|c|c|c|}
\hline $\begin{array}{c}\text { Epoch } \\
\text { gg-mm-yy }\end{array}$ & Target & $\begin{array}{c}\text { HPBW } \\
\text { mas } \times \text { mas, }\end{array}$ & $\begin{array}{c}\text { Noise } \\
\text { mJy/beam }\end{array}$ & $\begin{array}{l}S_{\text {tot }} \\
\text { mJy }\end{array}$ & $\begin{array}{r}\text { Core/jet } \\
\text { mJy }\end{array}$ & Notes \\
\hline $2005-06-22$ & $0034+25$ & $3.3 \times 1.6(-15)$ & 0.1 & 3.6 & 3.6 & $\bar{C}$ \\
\hline 2005-06-22 & $0055+26$ & $3.3 \times 1.6(-14)$ & 0.09 & 7.5 & 7.5 & $\mathrm{C}$ \\
\hline 2005-07-20 & $0149+35$ & $3.0 \times 1.4(0)$ & 0.08 & 3.2 & 3.2 & $\mathrm{C}$ \\
\hline 2005-07-20 & $0300+16$ & $3.2 \times 1.7(-1)$ & 0.1 & 6.1 & 6.1 & $\mathrm{C}$ \\
\hline 2005-07-20 & $0356+10$ & $3.3 \times 1.6(-4)$ & 0.12 & 2.2 & 2.2 & $\mathrm{C}$ \\
\hline $2005-07-20$ & $0708+32 B$ & $1.5 \times 1.5(-)$ & 0.08 & 10.5 & $\begin{array}{l}5.9 \\
4.6\end{array}$ & $\begin{array}{l}\text { double:A } \\
\text { double:B }\end{array}$ \\
\hline $2005-07-20$ & $0722+30$ & & 0.1 & ND & ND & - \\
\hline $2005-07-02$ & $0802+24$ & $3.5 \times 3.5(-)$ & 0.05 & 3.6 & $\begin{array}{l}2.1 \\
1.5\end{array}$ & $\begin{array}{l}\text { two-sided:C } \\
\text { two-sided:J }\end{array}$ \\
\hline 2005-07-02 & $0836+29-\mathrm{I}$ & $1.4 \times 1.4(-)$ & 0.08 & 47.8 & $\begin{array}{r}24.2 \\
5.8 \\
17.1\end{array}$ & $\begin{array}{l}\text { one-sided:C } \\
\text { one-sided:J1 } \\
\text { one-sided:J2 }\end{array}$ \\
\hline 2005-07-02 & $0838+32$ & $3.0 \times 1.7(12)$ & 0.09 & 7.0 & 7.0 & $\mathrm{C}$ \\
\hline 2005-06-17 & $0915+32$ & $2.9 \times 1.6(-6)$ & 0.1 & 13.6 & 13.6 & $\mathrm{C}$ \\
\hline 2005-07-02 & $1113+29$ & $4 \times 4(-)$ & 0.06 & 40.0 & $\begin{array}{r}36.8 \\
3.2\end{array}$ & $\begin{array}{l}\text { one-sided:C } \\
\text { one-sided:J }\end{array}$ \\
\hline 2005-07-02 & $1116+28$ & $3.3 \times 1.5(5)$ & 0.09 & 11.5 & 11.5 & $\mathrm{C}$ at $5 \mathrm{GHz}$ \\
\hline 2003-08-07 & $1116+28$ & $10.8 \times 5.5(28)$ & 0.13 & 9.5 & 9.5 & $\mathrm{C}$ at $1.6 \mathrm{GHz}$ \\
\hline 2005-06-17 & $1122+39$ & $2.6 \times 1.8(-1)$ & 0.10 & 8.8 & 8.8 & $\mathrm{C}$ \\
\hline 2005-06-17 & $1204+24$ & $3.0 \times 1.7(-4)$ & 0.09 & 5.3 & 5.3 & $\mathrm{C}$ \\
\hline $2005-06-17$ & $1251+27 B$ & $2 \times 2(-)$ & 0.06 & 10.4 & $\begin{array}{l}6.2 \\
4.2\end{array}$ & $\begin{array}{l}\text { two-sided:C } \\
\text { two-sided:J }\end{array}$ \\
\hline $2005-06-17$ & $1319+42$ & $2.3 \times 1.4(0)$ & 0.1 & 5.0 & $\begin{array}{l}3.0 \\
2.0\end{array}$ & $\begin{array}{l}\text { two-sided:C } \\
\text { two-sided:J }\end{array}$ \\
\hline $2005-06-27$ & $1339+26$ & & 0.1 & ND & ND & - \\
\hline $2005-06-27$ & $1346+26$ & see Liuzzo et al. & & & & $\mathrm{Z}$ structure \\
\hline 2003-08-07 & $1350+31$ & $12 \times 10,(24)$ & 0.1 & 235 & $\begin{array}{r}17.2 \\
2.5 \\
2.0 \\
192 \\
11.9 \\
11.2\end{array}$ & $\begin{array}{c}\text { at } 1.6 \mathrm{GHz}: \mathrm{C} \\
\text { J } \\
\text { CJ } \\
\text { E1 see Sect. } 4.1 .2 \\
\text { E2 } \\
\text { E3 }\end{array}$ \\
\hline $2005-06-27$ & $1357+28$ & $3 \times 2(0)$ & 0.15 & 5.6 & $\begin{array}{l}3.8 \\
1.8\end{array}$ & $\begin{array}{l}\text { two-sided:C } \\
\text { two-sided:J }\end{array}$ \\
\hline 2003-08-07 & $1422+26$ & $10.1 \times 4.5($ & 0.15 & 9.3 & $\begin{array}{l}5.1 \\
4.2\end{array}$ & $\begin{array}{c}\text { one-sided:C? } 1.6 \mathrm{GHz} \\
\mathrm{J} \text { ? }\end{array}$ \\
\hline $2003-08-30$ & $1448+63$ & $9.8 \times 4.5(-5)$ & 0.15 & ND & ND & $1.6 \mathrm{GHz}$, see text \\
\hline $2005-06-17$ & $1502+26$ & $2 \times 2(-)$ & 0.06 & 7.8 & $\begin{array}{l}0.5 \\
3.1 \\
4.2\end{array}$ & $\begin{array}{l}\text { 5GHz two-sided:C } \\
\text { two-sided:E1 } \\
\text { two-sided:W1 }\end{array}$ \\
\hline 2003-08-07 & $1502+26$ & $10.2 \times 4.5(-20)$ & 0.2 & 27.1 & $\begin{array}{r}0.3 \\
6.0 \\
11.3 \\
4.1\end{array}$ & $\begin{array}{c}\text { C } 1.6 \mathrm{GHz} \\
\mathrm{W} \\
\mathrm{E} 1 \\
\mathrm{~N}\end{array}$ \\
\hline 2003-08-07 & $1529+24$ & $11.0 \times 11.0(-)$ & 0.1 & & & $1.6 \mathrm{GHz}$, see text \\
\hline 2003-08-30 & $1557+26$ & $9.8 \times 4.5(2)$ & 0.12 & 10.5 & 10.5 & C $1.6 \mathrm{GHz}$ \\
\hline 2003-08-30 & $1613+27$ & $9.9 \times 4.5(3)$ & 0.12 & 9.3 & 9.3 & C $1.6 \mathrm{GHz}$ \\
\hline $2005-06-27$ & $1615+35 B$ & $2.8 \times 1.8(-17)$ & 0.06 & 23.6 & $\begin{array}{r}17.1 \\
6.5\end{array}$ & $\begin{array}{l}\text { one-sided:C } \\
\text { one-sided:J }\end{array}$ \\
\hline 2003-08-07 & $1621+38$ & $7 \times 7(-)$ & 0.09 & 17.9 & $\begin{array}{r}15.5 \\
2.4\end{array}$ & $\begin{array}{l}\text { 1.6GHz,one-sided:C } \\
\text { one-sided:J }\end{array}$ \\
\hline $2005-06-27$ & $1637+29$ & $2.8 \times 1.8(-12)$ & 0.1 & 7.8 & 7.8 & $\mathrm{C}$ \\
\hline 2005-06-22 & $1736+32$ & $2.9 \times 1.2(-32)$ & 0.14 & 7.6 & 7.6 & $\mathrm{C}$ \\
\hline $2005-06-22$ & $1752+32 B$ & $2 \times 2(-)$ & 0.08 & 9.2 & $\begin{array}{l}6.1 \\
3.1\end{array}$ & $\begin{array}{l}\text { two-sided:C } \\
\text { two-sided:J }\end{array}$ \\
\hline 2005-06-22 & $2236+35$ & $3.3 \times 1.6(-19)$ & 0.09 & 8.8 & 8.8 & $\mathrm{C}$ \\
\hline
\end{tabular}

In Col. 7 (notes) we indicate the component for which flux density is reported in Col. 6 (core/jet). $\mathrm{C}=$ core component; $\mathrm{j}=$ jet. We also report the observing frequency when at $1.6 \mathrm{GHz}$, in all other cases the observations are at $5 \mathrm{GHz}$. 
E. Liuzzo et al.: The Bologna complete sample of nearby radio sources. II., Online Material $p 5$

Table 4. Parameters for observed sources.

\begin{tabular}{|c|c|c|c|c|c|c|c|c|}
\hline Name & $\begin{array}{l}\text { Type } \\
\text { Kpс }\end{array}$ & $\begin{array}{l}\text { Type } \\
\text { pc }\end{array}$ & $\begin{array}{c}S_{\mathrm{cVLBI}} \\
\mathrm{mJy}\end{array}$ & $\begin{array}{c}S_{\text {totVLBI }} \\
\text { mJy }\end{array}$ & $\begin{array}{c}S_{\mathrm{cVLA}} \\
\mathrm{mJy}\end{array}$ & $\begin{array}{c}S_{\text {totVLBI }} / S_{\text {cVLA }} \\
\%\end{array}$ & $\begin{array}{c}\mathrm{j} / \mathrm{cj} \text { ratio } \\
\%\end{array}$ & $\begin{array}{c}\text { Core } \\
\text { dominance }\end{array}$ \\
\hline $0034+25$ & FR I & $\bar{C}$ & 3.6 & 3.6 & 10 & 36 & $\ldots$ & 1.10 \\
\hline $0055+26$ & FR I & $\mathrm{C}$ & 7.5 & 7.5 & 11 & 68 & $\ldots$ & 0.26 \\
\hline $0055+30$ & FR I & two-sid & 296 & 477 & 588 & 81 & 42 & 8.71 \\
\hline $0104+32$ & FR I & one-sid & 72 & 90 & 92 & 98 & $>16$ & 0.60 \\
\hline $0106+13$ & FR II & two-sid & 11.0 & 30 & 24 & 100 & 2 & 0.21 \\
\hline $0116+31$ & $\mathrm{C}$ & $\mathrm{CSO}$ & 20.0 & 1250 & - & 100 & $\ldots$ & 1.07 \\
\hline $0149+35$ & FR I & $\mathrm{C}$ & 3.2 & 3.2 & 5 & 64 & $\ldots$ & 0.25 \\
\hline $0206+35$ & FR I & one-sid & 75 & 87 & 106 & 82 & $>14$ & 2.14 \\
\hline $0220+43$ & FR I & two-sid & 105 & 180 & 182 & 99 & 10 & 0.98 \\
\hline $0222+36$ & $\mathrm{C}$ & two-sid & 58.2 & 120 & 140 & 86 & $\ldots$ & 12.02 \\
\hline $0258+35$ & CSS & one-sid & 7.4 & 243 & $<243$ & 100 & $\ldots$ & $<2.75$ \\
\hline $0300+16$ & FR I & $\mathrm{C}$ & 6.1 & 6.1 & 10 & 61 & $\ldots$ & 0.07 \\
\hline $0326+39$ & FR I & one-sid & 55.5 & 74 & 78 & 95 & $>25$ & 1.91 \\
\hline $0331+39$ & $\mathrm{C}$ & one-sid & 90 & 104 & 149 & 70 & $>12$ & 3.24 \\
\hline $0356+10$ & FR II & $\mathrm{C}$ & 2.2 & 2.2 & 9 & 24 & $\ldots$ & 0.05 \\
\hline $0648+27$ & $\mathrm{C}$ & one-sid & 4.4 & 12.8 & 58 & 22 & $>8$ & 7.41 \\
\hline $0708+32 B$ & FR I & $\mathrm{CSO} ?$ & ? & 10.5 & 15 & 70 & $\ldots$ & 2.63 \\
\hline $0755+37$ & FR I & one-sid & 138 & 216 & 195 & 100 & $>20$ & 3.16 \\
\hline $0802+24$ & FR II & two-sid & 2.1 & 3.6 & 8 & 45 & 1 & 0.13 \\
\hline $0836+29-\mathrm{II}$ & FR I & one-sid & 146 & 167 & 131 & 100 & $>20$ & 8.32 \\
\hline $0836+29-\mathrm{I}$ & FR I & one-sid & 24.2 & 47.8 & 63 & 76 & $>50$ & $\ldots$ \\
\hline $0838+32$ & FR I & $\mathrm{C}$ & 7.0 & 7.0 & 7.5 & 93 & $\ldots$ & 0.41 \\
\hline $0844+31$ & FR II & two-sid & 27.0 & 32 & 40 & 80 & 1 & 1.35 \\
\hline $0915+32$ & FR I & $\mathrm{C}$ & 13.6 & 13.6 & 8.0 & 100 & $\ldots$ & 0.98 \\
\hline $1003+35$ & FR II & one-sid & 152.8 & 466 & 400 & 100 & $>45$ & 14.13 \\
\hline $1037+30$ & $\mathrm{C}$ & ND & ND & $<2$ & $<84$ & - & $\ldots$ & $<10$ \\
\hline $1040+31$ & $\mathrm{C}$ & two-sid & 37.6 & 48 & 55 & 87 & 5 & 2.00 \\
\hline $1101+38$ & BL Lac & one-sid & 335 & 448 & 640 & 70 & $>110$ & 24.55 \\
\hline $1102+30$ & FR I & two-sid & 14.3 & 17 & 26 & 65 & $1.7 ?$ & 2.29 \\
\hline $1113+29$ & FR I & one-sid & 36.8 & 40.0 & 41 & 98 & $>5$ & 0.89 \\
\hline $1116+28$ & FR I & $\mathrm{C}$ & 11.5 & 11.5 & 30 & 38 & $\ldots$ & 2.34 \\
\hline $1122+39$ & FR I & $\mathrm{C}$ & 8.8 & 8.8 & 6 & 100 & $\ldots$ & 0.18 \\
\hline $1142+20$ & FR I & one-sid & 125 & 172 & 200 & 86 & $>24$ & 1.17 \\
\hline $1144+35$ & FR I & two-sid & 50.1 & ? & - & - & $\ldots$ & .. \\
\hline $1204+24$ & FR I & $\mathrm{C}$ & 5.3 & 5.3 & 8 & 66 & $\ldots$ & 4.57 \\
\hline $1204+34$ & FR II & one-sid & 33.7 & 39 & 23 & 100 & $>2$ & 2.09 \\
\hline $1217+29$ & $\mathrm{C}$ & two-sid & 31.5 & 121 & 162 & 75 & $>2$ & $<2.75$ \\
\hline $1222+13$ & FR I & one-sid & 105 & 126 & 180 & 70 & $>10$ & 0.32 \\
\hline $1228+12$ & FR I & one-sid & 450 & 1600 & 4000 & 40 & $>150$ & 0.91 \\
\hline $1251+27 B$ & FR II & two-sid & 6.2 & 10.4 & 12 & 87 & 2 & 0.38 \\
\hline $1257+28$ & FR I & one-sid & 0.79 & 1.07 & 1.1 & 97 & $>2$ & 0.06 \\
\hline $1316+29$ & FR I & two-sid & 5.3 & 23 & 31 & 74 & 1 & 1.29 \\
\hline $1319+42$ & FR II & two-sid & 3.0 & 5.0 & 6 & 83 & 2 & 0.19 \\
\hline $1321+31$ & FR I & two-sid & 3.7 & 11 & 21 & 52 & $1 ?$ & 0.26 \\
\hline $1322+36$ & FR I & one-sid & 45 & 60 & 150 & 40 & $>20$ & 3.09 \\
\hline $1339+26$ & FR I & ND & - & - & $<55$ & - & $\ldots$ & $<4.07$ \\
\hline $1346+26$ & FR I & two-sid & 4.1 & 44 & 53 & 83 & $\ldots$ & 2.04 \\
\hline $1350+31$ & FR I & two-sid & 14.4 & 23 & - & - & 2 & $<1.42$ \\
\hline $1357+28$ & FR I & two-sid & 3.8 & 5.6 & 6.2 & 90 & $1 ?$ & 0.10 \\
\hline $1414+11$ & FR I & two-sid & 65.1 & 86 & 77 & 100 & 2 & 0.95 \\
\hline $1422+26$ & FR I & one-sid & 1.7 & 3 & 25.0 & 12 & $>20 ?$ & 0.81 \\
\hline $1448+63$ & FR I & C? & 1.1 & 2 & 29 & 7 & $\ldots$ & 0.48 \\
\hline $1502+26$ & FR I & two-sid & 0.5 & 7.8 & 80 & 10 & 1 & 0.78 \\
\hline $1521+28$ & FR I & one-sid & 39.5 & 44 & 40 & 100 & $>17$ & 2.88 \\
\hline $1529+24$ & FR II & $\mathrm{C}$ & 3.4 & 3 & 30 & 10 & $\ldots$ & 0.98 \\
\hline $1553+24$ & FR I & one-sid & 40.7 & 46 & 40 & 100 & $>20$ & 6.17 \\
\hline $1557+26$ & C & one-sid & 7.8 & 9 & 30 & 30 & $>4$ & 8.13 \\
\hline
\end{tabular}


E. Liuzzo et al.: The Bologna complete sample of nearby radio sources. II., Online Material p 6

Table 4. continued.

\begin{tabular}{ccccccccc}
\hline \hline Name & $\begin{array}{c}\text { Type } \\
\text { Kpc }\end{array}$ & $\begin{array}{c}\text { Type } \\
\text { pc }\end{array}$ & $\begin{array}{c}S_{\text {cVLBI }} \\
\text { mJy }\end{array}$ & $\begin{array}{c}S_{\text {totVLBI }} \\
\text { mJy }\end{array}$ & $\begin{array}{c}S_{\text {cVLA }} \\
\text { mJy }\end{array}$ & $\begin{array}{c}S_{\text {totVLBI }} / S_{\text {cVLA }} \\
\%\end{array}$ & $\begin{array}{c}\text { j/cj ratio } \\
\%\end{array}$ & $\begin{array}{c}\text { Core } \\
\text { dominance }\end{array}$ \\
\hline $1613+27$ & FR I & C & 7.8 & 8 & 25 & 32 & $\ldots$ & 3.31 \\
$1615+35 B$ & FR I & one-sid & 17.1 & 23.6 & 28 & 84 & $>15$ & 0.37 \\
$1621+38$ & FR I & one-sid & 12.9 & 20 & 50 & 40 & $>5$ & 2.29 \\
$1626+39$ & FR I & two-sid & 29 & 91 & 105 & 87 & 1.1 & 0.76 \\
$1637+29$ & FR I & C & 7.8 & 7.8 & 13 & 60 & $\ldots$ & 1.48 \\
$1652+39$ & BL-Lac & one-sid & 450 & 1000 & 1250 & 80 & $>1250$ & 64.54 \\
$1658+30$ & FR I & one-sid & 60.0 & 76 & 84 & 90 & $>8.5$ & 3.09 \\
$1736+32$ & FR I & C & 7.6 & 7.6 & 8 & 95 & $\ldots$ & 0.95 \\
$1752+32 B$ & FR I & two-sid & 6.1 & 9.2 & 12 & 77 & 2 & 1.41 \\
$1827+32$ A & FR I & one-sid & 5.9 & 15 & 26 & 58 & $>7.5$ & 2.51 \\
$1833+32$ & FR II & one-sid & 105 & 131 & 188 & 70 & $>20$ & 2.75 \\
$1842+45$ & FR II & one-sid & 34.0 & 52 & 62 & 84 & $>10$ & 1.23 \\
$1845+79$ & FR II & one-sid & $? ?$ & $? ?$ & 330 & - & $>100$ & 3.02 \\
$1855+37$ & C & ND & ND & $<2$ & $<100$ & - & $\ldots$ & $<7.94$ \\
$2116+26$ & FR I & two-sid & $?$ & $?$ & 47 & - & 1 & 2.63 \\
$2229+39$ & FR I & C & 25.9 & 30 & 37 & 81 & $\ldots$ & 0.35 \\
$2236+35$ & FR I & C & 8.8 & 8.8 & 8.0 & 100 & $\ldots$ & 0.39 \\
$2243+39$ & FR II & two-sid & 18 & 125 & 130 & 96 & 1.1 & 1.51 \\
$2335+26$ & FR I & one-sid & 168 & 234 & 270 & 87 & $>10$ & 1.82 \\
\hline
\end{tabular}

VLBI data are at $5 \mathrm{GHz}$. 